Series A

I. MATHEMATICA

488

\title{
TOPOLOGICAL AND METRIC PROPERTIES OF QUASIREGULAR MAPPINGS
}

BY

O. MARTIO, S. RICKMAN and J. VÄISÄLÄ 
Copyright (C) 1971 by

Academia Scientiarum Fennica

Communicated 11 December 1970 by Olli Lehto 


\section{Introduction}

1.1. This paper is a continuation to [13] and [14]. Our main interest is centered on the branch set $B_{f}$ of a quasiregular mapping $f: G \rightarrow R^{n}$. In Section 2 we consider the case $B_{f}=\varnothing$, which means that $f$ is a local homeomorphism. Section 3 deals with the properties of $f B_{f}$ in the case $B_{f} \neq \varnothing$. For example, we show that $\Lambda_{n-2}\left(f B_{f}\right)>0$ and consider the case where $f$ has an essential isolated singularity. In Section 4 we present relations between the local degree $i(x, f)$ and the dilatation $K(f)$. In Section 5 we improve some results of [13] concerning the linear dilatations.

1.2. Notation and terminology. We shall use the same notation and terminology as in [13] and [14]. All point sets are assumed to lie in the compactified $n$-space $\bar{R}^{n}=R^{n} \cup\{\infty\}$. Throughout the paper we assume $n \geqq 2$. The notation $f: G \rightarrow R^{n}$ or $f: G \rightarrow \bar{R}^{n}$ includes the assumptions that $G$ is a domain in $R^{n}$ or $\bar{R}^{n}$, respectively, and that $f$ is continuous. If $\Delta$ is a segment of line and if $\alpha: \Delta \rightarrow \bar{R}^{n}$ is a path, we let $|\alpha|$ denote the locus $\alpha \Delta$ of $\alpha$. More generally, if $c=\Sigma m_{i} \sigma_{i}$ is a singular 1-chain, then $|c|=U\left|\sigma_{i}\right|$. The inner product of two vectors $x, y$ is written as $(x \mid y)$.

\section{Local homeomorphisms}

2.1. In this section we show that a $K$-quasiregular local homeomorphism of the unit ball $B^{n}, n \geqq 3$, is homeomorphic in a smaller ball $B^{n}(r)$, where $r$ depends only on $n$ and $K$. This result is applied to give a sufficient condition for the equicontinuity of a family of $K$-quasiregular local homeomorphisms.

We need some topological results on local homeomorphisms. A set $Q \subset \bar{R}^{n}$ is said to be relatively locally connected if every point of $\bar{Q}$ has arbitrarily small neighborhoods $U$ such that $U \cap Q$ is connected.

2.2. Lemma. Suppose that $f: G \rightarrow \bar{R}^{n}$ is a local homeomorphism, that $Q$ is a simply connected and locally pathwise connected set in $\bar{R}^{n}$, and that $P$ is a component of $f^{-1} Q$ such that $\bar{P} \subset G$. Then $f$ maps $P$ homeo- 
morphically onto $Q . I f$, in addition, $Q$ is relatively locally connected, $f$ maps $\bar{P}$ homeomorphically onto $\bar{Q}$.

Proof. In order to prove the first statement of the theorem, it suffices to show that $f$ defines a covering mapping of $P$ onto $Q$ [9, p. 91]. If $x_{0} \in P$, every path in $Q$ starting at $f\left(x_{0}\right)$ may be uniquely lifted to a path in $P$ starting at $x_{0}$. Hence $f P=Q$. Let $y \in Q$, and let $P \cap f^{-1}(y)=$ $\left\{x_{1}, \ldots, x_{k}\right\}$. Choose disjoint neighborhoods $U_{i}$ of $x_{i}$ such that $U_{i} \subset G$ and $f$ is injective in each $U_{i}$. Then $F=\bar{P} \backslash\left(U_{1} \cup \cdots \cup U_{k}\right)$ is compact, and $y \notin f F$. Choose a neighborhood $V$ of $y$ such that $Q \cap V$ is connected and $V \subset\left(\cap f U_{i}\right) \backslash f F$. Then $P \cap f^{-1} V$ has exactly $k$ components $D_{1}, \ldots, D_{k}$, one in each $U_{i}$, and $f$ maps $D_{i}$ homeomorphically onto $Q \cap V$. Thus $f$ defines a covering mapping $P \rightarrow Q$.

Assume now that $Q$ is relatively locally connected. We must show that an arbitrary point $y \in \bar{Q}$ has exactly one pre-image in $\bar{P}$. Choose a sequence $V_{1} \supset V_{2} \supset \cdots$ of neighborhoods of $y$ such that $V_{j} \cap Q$ is connected for all $j$ and $\cap V_{j}=\{y\}$. It is casy to see that $\bar{P} \cap f^{-1}(y)=$ $\bigcap_{j=1}^{\infty} \bar{A}_{j}$ where $A_{j}=P \cap f^{-1} V_{j}$. Since $f$ defines a homeomorphism $P \rightarrow Q$, every $A_{j}$ is connected. Hence $\bar{A}_{1} \supset \bar{A}_{2} \supset \cdots$ is a nested sequence of compact connected sets, which implies that $\bar{P} \cap f^{-1}(y)$ is non-empty and connected. Since $f^{-1}(y)$ is discrete, $\bar{P} \cap f^{-1}(y)$ consists of exactly one point.

2.3 Theorem. If $n \geqq 3$ and if $f: B^{n} \rightarrow R^{n}$ is a $K$-quasiregular local homeomorphism, then $f$ is injective in a ball $B^{n}(\psi(n, K))$, where $\psi(n, K)$ is a positive number depending only on $n$ and $K$.

Proof. We may assume that $f(0)=0$. As in [13], we let $U(0, f, r)$ denote the component of $f^{-1} B^{n}(r)$ which contains the origin. Let $r_{0}$ be the least upper bound of all positive numbers $r$ such that $\bar{C}(0, f, r) \subset B^{n}$. Fix $r \in\left(0, r_{0}\right)$, and set $l^{*}=l^{*}(0, f, r), L^{*}=L^{*}(0, f, r)$ (for notation, see $[13,4.1])$. By 2.2, $f$ maps $\bar{U}(0, f, r)$ homeomorphically onto $\bar{B}^{n}(r)$. Thus $f$ is injective in $B^{n}\left(l^{*}\right)$. Hence it suffices to find a lower bound for $l^{*}$.

Let $l=l\left(0, f, l^{*}\right)$, and suppose $l<r$. Then $A=U(0, f, r) \backslash$ $\bar{U}(0, f, l)$ is a ring, and $f$ maps $A K$-quasiconformally onto the spherical ring $B^{n}(r) \backslash \bar{B}^{n}(l)$. Since both boundary components of $A$ meet the sphere $S^{n-1}\left(l^{*}\right)$, it follows from a well-known estimate $[26,11.7]$ that cap $A \geqq a_{n}>0$, where $a_{n}$ depends only on $n$. Thus

$$
a_{n} \leqq \operatorname{cap} A \leqq K \operatorname{cap} f A=K \omega_{n-1}(\log (r / l))^{1-n} .
$$


This gives an inequality

$$
r / l \leqq \alpha(n, K)
$$

which is true also in the case $r=l$.

We shall now make use of a method of Zorič [28] and Agard-Marden [1]. Choose $x_{0} \in \partial U(0, f, r)$ such that $\left|x_{0}\right|=L^{*}$, and set $y_{0}=f\left(x_{0}\right)$. Then $\left|y_{0}\right|=r$. For $t \in(r, r+l)$ and for $\varphi \in(0, \pi]$ let

$$
C(t, \varphi)=\left\{y|| y-y_{0} \mid=t,\left(y_{0}-y \mid y_{0}\right)>r t \cos \varphi\right\} \text {. }
$$

Thus $C(t, \varphi)$ is a spherical cap (possibly a punctured sphere), which is symmetric with respect to the line segment $J=\left\{s y_{0}|-l| r<s<0\right\}$ and meets $J$ at $z_{t}=(r-t) y_{0} / r$. Let $z_{t}^{*}$ be the unique point in $U(0, f, r)$ $\cap f^{-1}\left(z_{t}\right)$, and let $C^{*}(t, \varphi)$ be the $z_{t}^{*}$-component of $f^{-1} C(t, \varphi)$. Let $\varphi_{t}$ be the least upper bound of all $\varphi \in(0, \pi]$ such that $f$ maps $C^{*}(t, \varphi)$ homeomorphically onto $C(t, \varphi)$. We show that $C^{*}\left(t, \varphi_{t}\right)$ meets $S^{n-1}\left(L^{*}\right)$ for every $t \in(r, r+l)$. Suppose that this is false. Then $C^{*}\left(t, \varphi_{t}\right) \subset B^{n}\left(L^{*}\right)$ for some $t$. By 2.2, $f$ maps $\bar{C}^{*}\left(t, \varphi_{t}\right)$ homeomorphically onto $\bar{C}\left(t, \varphi_{t}\right)$. Note that for $n=2$, the proof breaks down here, since $C(t, \pi)$ is then not relatively locally connected. By [28, Remark 1, p. 422] or by Corollary $3.8, f$ is injective in a neighborhood of $\bar{C}^{*}\left(t, \varphi_{t}\right)$. In view of the definition of $\varphi_{t}$, this implies $\varphi_{t}=\pi$, which means that $\bar{C}^{*}\left(t, \varphi_{t}\right)$ is a topological sphere. The bounded component $D$ of $\mathbf{C} \bar{C}^{*}\left(t, \varphi_{t}\right)$ is contained in $B^{n}\left(L^{*}\right)$. Since $\partial f D \subset S^{n-1}\left(y_{0}, t\right), f D=B^{n}\left(y_{0}, t\right)$. Thus $D$ is a component of $f^{-1} B^{n}\left(y_{0}, t\right)$. By 2.2, $f$ maps $\bar{D}$ homeomorphically onto $\bar{B}^{n}\left(y_{0}, t\right)$. Since $z_{t}^{*} \in \bar{D} \cap \bar{U}(0, \dot{f}, r)$ and since $\bar{B}^{n}\left(y_{0}, t\right) \cap \bar{B}^{n}(r)$ is connected, it follows from [28, Remark 2, p. 422] that $f$ is injective in $\bar{D} \cup \bar{U}(0, f, r)$. Since $y_{0} \in f D$, this implies that $x_{0} \in D$, which is impossible, because $D \subset B^{n}\left(L^{*}\right)$. Thus $C^{*}\left(t, \varphi_{t}\right)$ meets $S^{n-1}\left(L^{*}\right)$ for all $t \in(r, r+l)$.

Set $V=\cup C\left(t, \varphi_{t}\right)$ and $V^{*}=\cup C^{*}\left(t, \varphi_{t}\right)$, where the unions are taken over $t \in(r, r+l)$. Arguing as in [28, p. 425] we see that $V^{*}$ and $V$ are domains and that $f$ maps $V^{*}$ homeomorphically onto $V$. For esch $t \in(r, r+l)$ choose a point $x_{t}^{*} \in C^{*}\left(t, \varphi_{t}\right) \cap S^{n-1}\left(L^{*}\right)$. Let $\Gamma(t)$ bo the family of all paths joining $x_{t}^{*}$ and $z_{t}^{*}$ in $C^{*}\left(t, \varphi_{t}\right)$, and set $\Gamma=\cup \Gamma(t)$. Since $\left|z_{t}^{*}\right| \leqq l^{*}$

$$
M(\Gamma) \leqq \omega_{n-1}\left(\log \left(L^{*} / l^{*}\right)\right)^{1-n}
$$

On the other hand, a well-known modulus estimate $[26,10.12]$ yields

$$
M(f \Gamma) \geqq b_{n} \log (1+l / r)
$$

where the positive constant $b_{n}$ depends only on $n$. Since $M(f \Gamma) \leqq K M(T)$, we obtain from (2.4), (2.5), and (2.6) an inequality $l^{*} \geqq L^{*} \psi(n, K)$, where $\psi(n, K)>0$ depends only on $n$ and $K$. Since $L^{*} \rightarrow 1$ as $r \rightarrow r_{0}$, this proves the theorem. 
2.7. Corollary. Suppose that $n \geqq 3$, that $f: G \rightarrow R^{n}$ is a $K$-quasiregular mapping and that $x_{0} \in G \backslash B_{f}$. Then $f$ is injective in the ball $B^{n}\left(x_{0}, r\right)$ where $r=\psi(n, K) d\left(x_{0}, B_{f} \cup \partial G\right)$.

2.8. Corollary. (Theorem of Zoric [28]) If $n \geqq 3$ and if $f: R^{n} \rightarrow R^{n}$ is a quasiregular local homeomorphism, then $f$ is a homeomorphism.

2.9 Theorem. Suppose that $G$ is a domain in $\bar{R}^{n}, n \geqq 3$, that $K \geqq 1$. and that $r>0$. If $W$ is a family of $K$-quasimeromorphic local homeomorphisms $f: G \rightarrow \bar{R}^{n}$ such that every $f \in W$ omits two points $a_{f}, b_{f} \in \bar{R}^{n}$ with $q\left(a_{f}, b_{f}\right) \geqq r$, then $W$ is equicontinuous. Here $q$ is the spherical metric $[14,3.10]$.

Proof. For $f \in W$ let $T_{f}$ be a Möbius transformation such that $T_{f}\left(b_{f}\right)=\infty$. By 2.7, every point in $G$ has a neighborhood in which every $T_{f} \circ f$, and hence $f$, is injective. The theorem follows from the corresponding result for quasiconformal mappings [26, 19.2].

2.10. Corollary. If $W$ is a family of $K$-quasimeromorphic local homeomorphisms of a domain $G \subset \bar{R}^{n}, n \geqq 3$, and if every $f \in \Pi$ omits two fixed points in $\bar{R}^{n}$, then $W$ is equicontinuous.

2.11. Remark. The results $2.3,2.7,2.8,2.9$, and 2.10 fail to be true for $n=2$. The mappings $f_{j}(z)=e^{j z}$ serve as a counterexample in every case.

2.12. Path families and quasimeromorphic local homeomorphisms. Suppose that $f: G \rightarrow \bar{R}^{n}$ is quasimeromorphic and that $\Gamma$ is a path family in $G$. In [13] we conjectured that

$$
M(f \Gamma) \leqq K_{I}(f) M(\Gamma) .
$$

We shall now prove this inequality for local homeomorphisms. This result is needed in Section 3.

2.14. Lemma. If $f: G \rightarrow \bar{R}^{n}$ is a quasimeromorphic local homeomorphism and if $\Gamma$ is a path family in $G$, then (2.13) is true.

Proof. Since the family of all paths through a given point is of modulus zero, we may assume that $\infty \notin G$ and $\infty \notin f G$. We cover $G$ with a countable number of domains $U_{i}$ such that $\bar{U}_{i} \subset G$ and such that $f$ defines a homeomorphism $f_{i}: U_{i} \rightarrow f U_{i}$. Set $g_{i}=f_{i}^{-1}$. Let $\Gamma^{*}$ be the family of all paths $\gamma \in \Gamma$ such that $f \circ \gamma$ is locally rectifiable. Let $\Gamma_{i}$ be the family 
of all paths $\gamma \in T^{*}$ which have a closed subpath $\beta$ such that $|\beta| \subset U_{i}$ and such that $g_{i}$ is not absolutely continuous on $f \circ \beta$. By Fuglede's theorem [26, 28.2] $M\left(f \Gamma_{i}\right)=0$. Setting $\Gamma_{0}=\Gamma^{*} \backslash\left(\cup \Gamma_{i}\right)$ we thus have $M\left(f \Gamma_{0}\right)=M(f T)$. Hence it suffices to show that

$$
M\left(f \Gamma_{0}\right) \leqq K_{I}(f) M(\Gamma) .
$$

Let $E$ be the set of all $x \in G$ such that $f$ is differentiable at $x$ and $J(x, f)>0$. Then $E$ is a Borel set and $m(G \backslash E)=0$. Suppose that $\varrho \in F(\Gamma)$. Define $\sigma: G \rightarrow \dot{R}^{1}$ by

$$
\begin{array}{ll}
\sigma(x)=\varrho(x) / l\left(f^{\prime}(x)\right) & \text { for } x \in E, \\
\sigma(x)=\infty & \text { for } x \in G \backslash E .
\end{array}
$$

Then $\sigma$ is a Borel function. Next define $Q^{\prime}: R^{n} \rightarrow \dot{R}^{1}$ by

$$
\begin{array}{ll}
\varrho^{\prime}(y)=\sup _{x \in f^{-1}(y)} \sigma(x) & \text { for } y \in f G, \\
\varrho^{\prime}(y)=0 & \text { for } y \in \mathbf{C} f G .
\end{array}
$$

Then

$$
\varrho^{\prime}(y) \geqq \sigma\left(g_{i}(y)\right) \geqq \varrho\left(g_{i}(y)\right) L\left(y, g_{i}\right)
$$

for $y \in f U_{i}$, where $L\left(y, g_{i}\right)=\lim \sup \left|g_{i}(y+h)-g_{i}(y)\right| / h \mid$, cf. [13, p. 16]. We shall show that $\varrho^{\prime} \in F\left(f \Gamma_{0}\right)$.

Since

$$
\left\{y^{\prime} \varrho^{\prime}(y)>t\right\}=\bigcup_{i=1}^{\infty}\left\{y \in f U_{i} \mid \sigma\left(g_{i}(y)\right)>t\right\}
$$

for all $t>0, Q^{\prime}$ is a Borel function. Suppose that $\gamma \in T_{0}$. Let $\varepsilon>0$, and let $\gamma_{\varepsilon}:[a, b] \rightarrow G$ be a closed subpath of $\gamma$ such that

$$
\int_{\gamma_{\varepsilon}} \varrho d s>1-\varepsilon .
$$

Divide $[\mathrm{a}, \mathrm{b}]$ into non-overlapping subintervals $\left[a_{0}, a_{1}\right], \ldots,\left[a_{k-1}, a_{k}\right]$ such that each $\gamma_{j}=\gamma \mid\left[a_{j-1}, a_{j}\right]$ is a path in some $U_{i}$. Then $(2.15)$ and a transformation formula $[26,5.3]$ imply

$$
\int_{f \circ \gamma_{j}} \varrho^{\prime} d s \geqq \int_{f \circ \gamma_{j}} \varrho\left(g_{i}(y)\right) L\left(y, g_{i}\right)|d y| \geqq \int_{\gamma_{j}} \varrho d s .
$$

Summing over $j$ yields

$$
\int_{f \circ \gamma} \varrho^{\prime} d s \geqq \int_{f \circ \gamma_{\varepsilon}} \varrho^{\prime} d s \geqq \int_{\gamma_{\varepsilon}} \varrho d s>1-\varepsilon .
$$


Since $\varepsilon$ was arbitrary, this implies

$$
\int_{f \circ \gamma} \varrho^{\prime} d s \geqq 1 .
$$

Hence $\varrho^{\prime} \in F\left(f \Gamma_{0}\right)$. We thus obtain the inequality

$$
M\left(f \Gamma_{0}\right) \leqq \int \varrho^{\prime n} d m
$$

Choose an increasing sequence of Borel functions $\varrho_{j}: R^{n} \rightarrow R^{1}$ such that either $0=\varrho_{j}(y)=\varrho^{\prime}(y)$ or $0<\varrho_{j}(y)<\varrho^{\prime}(y)$ for all $y \in R^{n}$ and such that $\varrho_{j}(y) \rightarrow \varrho^{\prime}(y)$ for all $y \in R^{n}$. Let $A_{j}$ be the set of all $x \in G$ such that $\varrho_{j}(f(x)) \leqq \sigma(x)$. From the definition of $\varrho^{\prime}$ it follows that $f A_{j}=f G$. In other words, $N\left(y, f, A_{j}\right) \geqq 1$ for all $y \in f G$. Using a transformation formula in [16, Theorem 3, p. 364] we obtain

$$
\begin{aligned}
\int \varrho_{j}^{n} d m & \leqq \int \varrho_{j}(y)^{n} N\left(y, f, A_{j}\right) d m(y) \\
& =\int \varrho_{A_{j}}(f(x))^{n} J(x, f) d m(x) \\
& \leqq K_{I}(f) \int_{A_{j}} \sigma(x)^{n} l\left(f^{\prime}(x)\right)^{n} d m(x) \\
& \leqq K_{I}(f) \int \varrho^{n} d m .
\end{aligned}
$$

Letting $j \rightarrow \infty$ and using (2.16) we obtain

$$
M\left(f \Gamma_{0}\right) \leqq K_{I}(f) \int \varrho^{n} d m
$$

This proves (2.13).

\section{Properties of $f B_{f}$}

First we prove a relation between Hausdorff measure and simple connectedness. This is used to show that $\Lambda_{n-2}\left(f B_{f}\right)>0$ for a discrete and open mapping $f: G \rightarrow \bar{R}^{n}$ with $B_{f} \neq \varnothing$. Next we examine the behavior of a quasimeromorphic mapping $f$ with an isolated essential singularity. We prove that the set of asymptotic values and $\mathbf{C} f G$ are contained in the closure of $f B_{f}$. We also give an outline of a proof for Iversen's theorem which states that $\mathbf{C} f G$ is contained in the set of asymptotic values. In the 
rest of the section we consider for $n=3$ the case where $f B_{f}$ is contained in a tamely embedded arc.

3.1. Lemma. Let $T \subset R^{n}$ be a 2-dimensional plane and let $A \subset R^{n}$ be such that $\Lambda_{n-2}(A)=0$. Then $m(A+T)=0$.

Proof. It is sufficient to show that $m(A+Q)=0$ whenever $Q \subset T$ is a closed square. Let $\varepsilon>0$. Denote by $h$ the length of a side of $Q$. Choose a covering of $A$ by balls $B_{i}=B^{n}\left(x_{i}, r_{i}\right)$ such that $r_{i}<1$ and $\sum r_{i}^{n-2}<\varepsilon$. Then $A+Q \subset \cup\left(B_{i}+Q\right)$. On the other hand,

$$
m\left(B_{i}+Q\right) \leqq\left(h+2 r_{i}\right)^{2} 2^{n-2} r_{i}^{n-2}<(h+2)^{2} 2^{n-2} r_{i}^{n-2},
$$

hence

$$
m(A+Q) \leqq \sum m\left(B_{i}+Q\right) \leqq(h+2)^{2} 2^{n-2} \varepsilon,
$$

and the lemma follows.

3.2. Lemma. Let $A \subset R^{n}$ be such that $\Lambda_{n-2}(A)=0$, and let $E \subset R^{n}$ be a set which has a countable covering by 2-dimensional planes $T_{1}, T_{2}, \ldots$ Then $(E+y) \cap A=\varnothing$ for almost every $y \in R^{n}$.

Proof. Denote $H=\left\{y \in R^{n} \mid(E+y) \cap A \neq \emptyset\right\}$. If $y \in H$, there exists $z \in E$ such that $z+y \in A$. Hence $y \in A-z$, and so $y \in A-E$. This yields $H \subset A-E \subset \cup\left(A-T_{i}\right)$. Since 3.1 implies $m\left(A-T_{i}\right)=0$, we have $m(H)=0$. The lemma is proved.

The next result is perhaps well-known, but the authors have been unable to find it in literature.

3.3. Lemma. Let $G \subset R^{n}$ be a simply connected domain and let $A$ be closed in $G$ such that $\Lambda_{n-2}(A)=0$. Then $G \backslash A$ is simply connected.

Proof. Suppose that $\gamma: I \rightarrow G \backslash A, I=[0,1]$, is a path with $\gamma(0)=$ $\gamma(1)=x_{0}$. By assumption there exists a homotopy $h: I^{2} \rightarrow G$ such that $h(0, t)=\gamma(t), h(1, t)=h(s, 0)=h(s, 1)=x_{0}$ for all $(s, t) \in I^{2}$. Let $\tau$ be a triangulation of $I^{2}$ and let $h_{1}: I^{2} \rightarrow G$ be a simplicial approximation of $h$ with respect to $\tau$. Then $h_{1}(1, t)=h_{1}(s, 0)=h_{1}(\mathrm{~s}, 1)=x_{0}$ for $(s, t) \in I^{2}$. Set $\gamma_{1}(t)=h_{1}(0, t)$. If the triangulation $\tau$ is sufficiently dense, then $(s, t) \mapsto s \gamma(t)+(1-s) \gamma_{1}(t)$ defines a homotopy $\gamma \simeq \gamma_{1}$ in $G \backslash A$. By 3.2 there exists $y \in R^{n}$ such that $|y|<\min \left(d\left(h_{1} I^{2}, \partial G\right), d\left(\left|\gamma_{1}\right|, \partial(G \backslash A)\right)\right)$ and $\left(h_{1} I^{2}+y\right) \cap A=\varnothing$. Thus we obtain the following homotopies in $G \backslash A: \gamma_{1} \simeq \gamma_{1}+y$ defined by $(s, t) \mapsto \gamma_{1}(t)+s y$ and $\gamma_{1}+y \simeq x_{0}+y$ 
defined by $(s, t) \mapsto h_{1}(s, t)+y$. This implies $\gamma \simeq x_{0}+y$ in $G \backslash A$, and the lemma follows.

3.4. Theorem. Let $f: G \rightarrow \bar{R}^{n}$ be discrete and open. If $B_{f} \neq \varnothing$, then $A_{n-2}\left(f B_{f}\right)>0$.

Proof. If $n=2$, the theorem is trivial. Suppose $n \geqq 3$. Let $x \in B_{f}$ and pick $r>0$ such that $U=U(x, f, r)$ is a normal neighborhood of $x$. The mapping $f \mid U$ as a pseudo-covering map in the sense of Church and Hemmingsen [4, p. 529]. Hence [4, Corollary 5.2] implies that $B^{n}(f(x), r) \backslash$ $f\left(B_{f} \cap U\right)$ is not simply connected. Since $f\left(B_{f} \cap U\right)$ is closed in $B^{n}(f(x), r)$, Lemma 3.3 yields $A_{n-2}\left(f B_{f}\right)>0$.

3.5 Remark. It has been conjectured [3, p. 368] that if $f: G \rightarrow R^{n}$ is discrete and open, then either $B_{f}=\varnothing$ or $\operatorname{dim} B_{f}=n-2$. For $n=3$ this has been proved by Trohimčuk [25]; for $n \geqq 4$ this is an open question. Since $\operatorname{dim} B_{f}=\operatorname{dim} f B_{f} \quad[4,2.2]$ and since $\operatorname{dim} A=k$ implies $\Lambda_{k}(A)>0$ $[10$, p. 104], Theorem 3.4 is a weaker result.

3.6. We next study the set $f B_{f}$ in the case where $f$ has an essential isolated singularity. For terminology, see [14, 4.2]. It turns out that there are striking differences between the cases $n=2$ and $n \geqq 3$.

We prove first some topological lemmas. Suppose that $f: G \rightarrow \bar{R}^{n}$ is sense-preserving, discrete, and open. If $C$ is a set such that $\bar{C} \subset G$ and if $y \in \bar{R}^{n}$, then the set $A=f^{-1}(y) \cap C$ is finite, and we set

$$
M(y, f, C)=\sum_{x \in A} i(x, f)
$$

(cf. Martio $[12,3.5])$. Moreover, we set $M *(f, C)=\sup M(y, f, C)$ over $y \in \bar{R}^{n}$. Then $N(f, C) \leqq M^{*}(f, C)$. Furthermore, covering $C$ with a finite number of normal domains $D_{1}, \ldots, D_{k}$ we see that $M^{*}(f, C) \leqq$ $\Sigma \mu\left(f, D_{j}\right)<\infty$.

3.7. Lemma. Suppose that $f: G \rightarrow \bar{R}^{n}$ is sense-preserving, discrete, and open, and that $F$ is a compact set in $G$. Then there is a neighborhood $U$ of $F$ such that $\bar{U} \subset G$ and $M^{*}(f, U)=M^{*}(f, F)$.

Proof. Suppose that the lemma is not true. Set $k=M^{*}(f, F)$. For each positive integer $j$, we can find a point $y_{j} \in \bar{R}^{n}$ and a finite set $A_{j} \subset f^{-1}\left(y_{j}\right)$ such that $d(x, F)<1 / j$ for $x \in A_{j}$ and such that $\Sigma i(x, f) \geqq k+1$ over $x \in A_{j}$. Passing to a subsequence we may assume that card $A_{j}=h$ is independent of $j$. Moreover, we may assume that $A_{j}=\left\{a_{j}^{1}, \ldots, a_{j}^{h}\right\}$ 
such that every sequence $a_{1}^{m}, a_{2}^{m}, \ldots$ converges to a point $a_{0}^{m} \in F$. It follows that $f\left(a_{0}^{m}\right)=y_{0}=\lim y_{j}$. Let $b_{1}, \ldots, b_{s}$ be the distinct points in $\left\{a_{0}^{1}, \ldots, a_{0}^{h}\right\}$, and let $Q_{r}=\left\{m \mid a_{0}^{m}=b_{r}\right\}, 1 \leqq r \leqq s$. Choose disjoint normal neighborhoods $U_{r}$ of $b_{r}, 1 \leqq r \leqq s$. Then there is $j$ such that $y_{j} \in f U_{1} \cap$ $\ldots \cap f U_{s}$ and such that $a_{j}^{m} \in U_{r}$ for all $m \in Q_{r}$ and $1 \leqq r \leqq s$. We obtain

$$
\begin{gathered}
k \geqq M\left(y_{0}, f, F\right) \geqq \sum_{r=1}^{s} i\left(b_{r}, f\right)=\sum_{r=1}^{s} \mu\left(y_{0}, f, U_{r}\right)= \\
\sum_{r=1}^{s} \mu\left(y_{j}, f, U_{r}\right) \geqq \sum_{r=1}^{s} \sum_{m \in Q_{r}} i\left(a_{j}^{m}, f\right)=\sum_{x \in A_{j}} i(x, f) \geqq k+1 .
\end{gathered}
$$

This contradiction proves the lemma.

3.8. Corollary. (Zoric $\left[28\right.$, p. 422]) Suppose that $f: G \rightarrow \bar{R}^{n}$ is a local homeomorphism and that $F$ is a compact set in $G$ such that $f \mid F$ is injective. Then $f$ is injective in some neighborhood of $F$.

3.9. Lemma. Suppose that $f: G \rightarrow \bar{R}^{n}$ is a discrete open mapping and that $U$ is a normal domain of $f$ such that $f U$ is relatively locally connected (see 2.1). If $y \in f\left(\partial U \backslash B_{f}\right)$, then

$$
N\left(y, f, \partial U \backslash B_{f}\right)=N\left(f, \partial U \backslash B_{f}\right)=N(f, \partial U)=N(f, U) .
$$

Proof. We may assume that $f$ is sense-preserving. Let $y \in f\left(\partial U \backslash B_{f}\right)$ and let $x_{1}, \ldots, x_{k}$ be the points of $f^{-1}(y) \cap\left(\partial U \backslash B_{f}\right)=f^{-1}(y) \cap \bar{U}$. Thus $k=N\left(y, f, \partial U \backslash B_{f}\right)$. Choose disjoint neighborhoods $U_{i}$ of $x_{i}$ such that $f \mid \bar{U}_{i}$ is injective, and choose then a neighborhood $V$ of $y$ such that $V \subset \cap f U_{i}$ and such that $V \cap f U=D$ is connected. By [13, 2.6], $f$ maps every component $C$ of $U \cap f^{-1} D$ onto $D$. Hence $y \in f \bar{C}$, which implies that the components of $U \cap f^{-1} D$ are the domains $C_{i}=U_{i} \cap f^{-1} D$. Choose $y_{1} \in D$. Using $[13,2.12]$ we obtain

$$
\begin{aligned}
N(f, U) & =\mu\left(y_{1}, f, U\right)=\sum_{i=1}^{k} \mu\left(y_{1}, f, C_{i}\right)=k=N\left(y, f, \partial U \backslash B_{f}\right) \\
& \leqq N\left(f, \partial U \backslash B_{f}\right) \leqq N(f, \partial U) .
\end{aligned}
$$

It remains to show that $N(f, \partial U) \leqq N(f, U)$. Pick $z \in \partial f U$ such that $N(z, f, \partial U)=N(f, \partial U)=h$. Let $\left\{x_{1}, \ldots, x_{h}\right\}=f^{-1}(z) \cap \partial U=f^{-1}(z) \cap \bar{U}$. Choose disjoint neighborhoods $U_{i}$ of $x_{i}$, set $F=\bar{U} \backslash\left(U_{1} \cup \ldots \cup U_{h}\right)$, and choose then a neighborhood $V$ of $z$ such that $V \cap f F=\varnothing$ and such that $V \cap f U=D$ is connected. Then each $U_{i}$ contains a component $C_{i}$ of $U \cap f^{-1} D$, and $f C_{i}=D$. Hence every point $z_{1} \in D$ has at least $h$ pre-images in $U$. Thus $N(f, U) \geqq N\left(z_{1}, f, U\right) \geqq h=N(f, \partial U)$. The lemma is proved. 
3.10. Lemma. (cf. Agard-Marden [1, 3.A]) Suppose that $f: G \rightarrow \bar{R}^{n}$ is light, that $A \subset f G$, and that $s: A \rightarrow G$ is a continuous section of $f$, that is, $f \circ s=\mathrm{id}$. If $A$ is relatively locally connected at a point $y \in \bar{A}$ (see 2.1), then the cluster set $C(s, y)$ is either a compact connected set in $\partial G$ or consists of a single point in $G$.

Proof. Choose a basic system $U_{1} \supset U_{2} \supset \ldots$ of neighborhoods of $y$ such that the sets $D_{j}=U_{j} \cap A$ are connected. Then the sets $s D_{j}$ are connected, which implies that $C(s, y)=\Omega \overline{s D_{j}}$ is a compact connected set in $\bar{G}$. If $x \in G \cap C(s, y)$, then $f(x)=y$ by the continuity of $f$. Thus $G \cap C(s, y) \subset f^{-1}(y)$. Since $f^{-1}(y)$ is totally disconnected, either $G \cap C(s, y)=\varnothing$ or $G \cap C(s, y)=C(s, y)$ consists of a single point.

3.11. Path lifting. The path lifting problem for light open mappings has been considered by Stoïlow [23, p. 354], [24, p. 109], Whyburn [27, p. 186], Floyd [5, p. 574], and by us $[13,2.7]$. We remark that our result $[13,2.7]$ is a direct corollary of Floyd's theorem, which was unfortunately overlooked in [13]. We shall now give the global version of this result.

Let $f: G \rightarrow \bar{R}^{n}$ be a mapping, let $\beta:[a, b) \rightarrow \bar{R}^{n}$ be a path, and let $x_{0} \in f^{-1}(\beta(a))$. We say that a path $\alpha:[a, c) \rightarrow G$ is a maximal lifting of $\beta$ starting at $x_{0}$ if:

(1) $\alpha(\alpha)=x_{0}$.

(2) $f \circ \alpha=\beta \mid[a, c)$.

(3) If $c<c^{\prime} \leqq b$, then there does not exist a path $\alpha^{\prime}:\left[a, c^{\prime}\right) \rightarrow G$ such that $\alpha=\alpha^{\prime} \mid[a, c)$ and $f \circ x^{\prime}=\beta \leqslant\left[a, c^{\prime}\right)$.

Similarly, we define the maximal lifting of a path $\beta:(a, b] \rightarrow \bar{R}^{n}$ terminating at a point $x_{0} \in f^{-1}(\beta(b))$.

If $\beta:[a, b) \rightarrow \bar{R}^{n}$ is a path and if $C \subset \bar{R}^{n}$, we say that $\beta(t) \rightarrow C$ as $t \rightarrow b$ if the spherical distance $q(\beta(t), C) \rightarrow 0$.

3.12. Lemma. Suppose that $f: G \rightarrow \bar{R}^{n}$ is light and open, that $x_{0} \in G$, and that $\beta:[a, b) \rightarrow \bar{R}^{n}$ is a path such that $\beta(a)=f\left(x_{0}\right)$ and such that either $\lim _{t \rightarrow b} \beta(t)$ exists, or $\beta(t) \rightarrow \partial f G$ as $t \rightarrow b$. Then $\beta$ has a maximal lifting $\alpha:[a, c) \rightarrow G$. If $\alpha(t) \rightarrow x_{1} \in G$ as $t \rightarrow c$, then $c=b$ and $f\left(x_{1}\right)=$ $\lim \beta(t)$. Otherwise $\alpha(t) \rightarrow \partial G$ as $t \rightarrow c$. If $f$ is discrete and if the local degree $i(\alpha(t), f)$ is constant for $t \in[a, c)$, then $\alpha$ is the only maximal lifting of $\beta$ starting at $x_{0}$.

Proof. Let $P$ be the set of all pairs $(\alpha, c)$ such that $a<c \leqq b$ and $\alpha:[a, c) \rightarrow G$ is a path satisfying the conditions (1) and (2) in 3.11 . From $[13,2.7]$ it follows that $P \neq \varnothing$. Define an ordering in $P$ as follows: 
$(\alpha, c) \leqq\left(\alpha^{\prime}, c^{\prime}\right)$ if $c \leqq c^{\prime}$ and $\alpha=\alpha^{\prime} \mid[a, c)$. By Zorn's lemma it is easy to see that $P$ contains a maximal element $(\alpha, c)$. Then $\alpha$ is a maximal lifting of $\beta$ starting at $x_{0}$.

Suppose that $\alpha(t) \rightarrow x_{1} \in G$ as $t \rightarrow c$. Since $f$ is continuous, $\beta(t)=$ $f(\alpha(t)) \rightarrow f\left(x_{1}\right)$. If $c<b$, we can use $[13,2.7]$ to construct a lifting $\left(\alpha^{\prime}, c^{\prime}\right)>$ $(\alpha, c)$. Hence $c=b$.

Next assume that $\alpha(t)$ does not tend to a point $x_{1} \in G$ as $t \rightarrow c$. We must show that $\alpha(t) \rightarrow \partial G$. Suppose that this is not true. Then there is a compact set $F \subset G$ and a sequence $t_{1}<t_{2}<\ldots$ such that $t_{j} \in[a, c)$, $t_{j} \rightarrow c$, and $\alpha\left(t_{j}\right) \in F$. We may assume that there is $x_{1} \in F$ such that $x\left(t_{j}\right) \rightarrow x_{1}$. Since $\alpha(t) \rightarrow x_{1}$, there is a neighborhood $U$ of $x_{1}$ and a sequence $\left(s_{j}\right)$ such that $\bar{U} \subset G, t_{j}<s_{j}<c$ and $\alpha\left(s_{j}\right) \notin U$. We may assume that $x(s) \in U$ for $s \in\left[t_{j}, s_{j}\right)$. Let $C_{j}$ be the continuum $\alpha\left[t_{j}, s_{j}\right] \subset \bar{U}$. Then $x_{1} \in \lim \inf C_{j}$. By [8,2-101, p. 101], $\lim \sup C_{j}=C$ is connected. If $\beta(t)$ tends to a point $y$ as $t \rightarrow c$, then $f C=\{y\}$. Since $f$ is light, $C=\left\{x_{1}\right\}$. This is impossible, because each $C_{j}$ meets $\partial U$. Hence $\beta(t)$ has no limit as $t \rightarrow c$. By the hypothesis of the theorem, $c=b$ and $\beta(t) \rightarrow \partial f G$ as $t \rightarrow b$. Hence there is $s<b$ such that $\beta(t) \notin f F$ for $t \in(s, b)$. On the other hand, $\beta\left(t_{j}\right)=f\left(\alpha\left(t_{j}\right)\right) \in f F$. This contradiction shows that $\lambda(t) \rightarrow \partial G$ as $t \rightarrow c$.

Now suppose that $f$ is discrete and that $i(\alpha(t), f)=k$ is constant for $t \in[a, c)$. We may assume that $f$ is sense-preserving. Let $\alpha^{\prime}:\left[\alpha, c^{\prime}\right) \rightarrow G$ be another maximal lifting of $\beta$ starting at $x_{0}$. Suppose that $\alpha \neq \alpha^{\prime}$. Then $E=\left\{t ; x(t) \neq \alpha^{\prime}(t)\right\} \neq \emptyset$. Set $v=\inf E$. Since $a \notin E, E$ is an open set in $(a, c)$. Hence $v \notin E$. Choose a normal neighborhood $V$ of $\alpha(v)=\alpha^{\prime}(v)$. Then there is $t \in(v . c)$ such that $\alpha(t) \in V, \alpha^{\prime}(t) \in V$ and $x(t) \neq \alpha^{\prime}(t)$. Then

$$
\begin{aligned}
k=\mu(f, V)= & \sum_{i\left(\alpha^{\prime}(t), f\right) \geqq k+i\left(\lambda^{\prime}(t), f\right) .}\left\{i(x, f) \operatorname{I}^{\prime} \cap f^{-1}(\beta(t))\right\} \geqq i(x(t), f)+
\end{aligned}
$$

This contradiction completes the proof of the lemma.

3.13. Definition. If $f: G \rightarrow \bar{R}^{n}$ is a mapping, a point $z \in \bar{R}^{n}$ is said to be an asymptotic value of $f$ at a boundary point $b \in \partial G$ if there is a path $x:[0,1) \rightarrow G$ such that $\alpha(t) \rightarrow b$ and $f(x(t)) \rightarrow z$ as $t \rightarrow 1$.

3.14. Theorem. Suppose that $b$ is an essential isolated singularity of a quasimeromorphic mapping $f: G \rightarrow \bar{R}^{n}, n \geqq 3$. If $z$ is an asymptotic value of $f$ at $b$, then $z \in \overline{f\left(B_{f} \cap U\right)}$ for every neighborhood $U$ of $b$.

Proof. Assume that there is a neighborhood $U$ of $b$. such that $z$ does not belong to the closure of $f\left(B_{f} \cap U\right)$. Using the methods of Zorič [28] and Agard-Marden [1] we show that this leads to a contradiction. 
We may assume that $b=0=z$. Pick $r_{0}>0$ such that $\bar{B}^{n}\left(r_{0}\right) \subset$ $U \cap(G \cup\{0\})$ and such that $S^{n-1}\left(r_{0}\right) \cap f^{-1}(0)=\varnothing$. Set $U_{0}=B^{n}\left(r_{0}\right) \backslash\{0\}$ and $g=f \mid U_{0}$. Choose $r^{\prime}>0$ such that $\bar{B}^{n}\left(r^{\prime}\right)$ does not meet $f S^{n-1}\left(r_{0}\right)$ $\cup g B_{g}$. Since 0 is an asymptotic value, there is a path $\alpha:[0,1) \rightarrow U_{0}$ such that $\alpha(t) \rightarrow 0$ and $\beta(t)=f(\alpha(t)) \rightarrow 0$ as $t \rightarrow 1$. We may assume that $0<|\beta(t)|<r^{\prime}$ for all $t \in(0,1)$. Thus $|\alpha| \subset U_{0} \backslash B_{g}$. For $0 \leqq t<1$ and $0<\varphi \leqq \pi$ we define the spherical caps

$$
C(t, \varphi)=\left\{\left.y \in R^{n}|| y|=| \beta(t)|,(y \mid \beta(t))>| y\right|^{2} \cos \varphi\right\} .
$$

Let $C^{*}(t, \varphi)$ be the $\alpha(t)$-component of $g^{-1} C(t, q)$, and let $\varphi_{t}$ be the least upper bound of all $\varphi \in(0, \pi]$ such that $g$ maps $C^{*}(t, \varphi)$ homeomorphically onto $C(t, \varphi)$. Set $C(t)=C\left(t, \varphi_{t}\right), C^{*}(t)=C^{*}\left(t, \varphi_{t}\right)$. Then $g$ defines a homeomorphism $g_{t}: C^{*}(t) \rightarrow C(t)$. We show that for almost every $r \in\left(0, r^{\prime}\right),|\beta(t)|=r$ implies $0 \notin \bar{C}^{*}(t)$.

If $0 \in \bar{C}^{*}(t)$, it follows from Lemma 3.10 that there is a point $y_{t} \in \bar{C}(t)$ such that $g_{t}^{-1}(y) \rightarrow 0$ as $y \rightarrow y_{t}$ in $C(t)$. Let $\Gamma(t)$ be the family of all paths which join $\beta(t)$ and $y_{t}$ in $C(t)$, and let $\Gamma^{*}(t)=g_{t}^{-1} \Gamma(t)$ and $\Gamma^{*}=-\cup \Gamma^{*}(t)$ over all $t$ such that $0 \in \bar{C}^{*}(t)$. Since all paths of $\Gamma^{*}$ converge to $0, M\left(\Gamma^{*}\right)=0$. By 2.14, this implies $M\left(g \Gamma^{*}\right)=0$. On the other hand, a well-known modulus estimate $[26,10.2]$ yields

$$
M\left(g \Gamma^{*}\right) \geqq b_{n} \int_{E} \frac{d r}{r}
$$

where $b_{n}>0$ depends only on $n$ and where $E=\left\{|\beta(t)| \mid 0 \in \bar{C}^{*}(t)\right\}$. Hence $m_{1}(E)=0$.

Let $T=\{t|0 \leqq t<1,| \beta(t) \mid \notin E\}$. If $t \in T$, then $\bar{C}^{*}(t) \subset U_{0} \backslash B_{g}$. From Lemma 2.2 it follows that $f$ maps $\bar{C}^{*}(t)$ homeomorphically onto $\bar{C}(t)$. By 3.8, $f$ is injective in a neighborhood of $\bar{C}^{*}(t)$. This is possible only if $\varphi_{t}=\pi$. Hence for every $t \in T, \bar{C}^{*}(t)=\bar{C}^{*}(t, \tau)$ is a topological sphere in $U_{0} \backslash B_{g}$, and $f$ maps $\bar{C}^{*}(t)$ homeomorphically onto $\bar{C}(t)=$ $S^{n-1}(|\beta(t)|)$. Let $D(t)$ be the bounded component of $\mathbf{C} \bar{C}^{*}(t)$. Let $T_{0}=$ $\{t \in T \mid 0 \in D(t)\}$. We divide the rest of the proof into two cases, according as 1 belongs to $\bar{T}_{0}$ or not.

Case 1. $1 \in \bar{T}_{0}$. We choose an increasing sequence of numbers $t_{j} \in T_{0}$ such that $t_{j} \rightarrow 1$. Let $r_{j}=\left|\beta\left(t_{j}\right)\right|$ and $D_{j}=D\left(t_{j}\right)$. Passing to a subsequence we may assume that $r_{j+1}<r_{j}$. Since $\alpha\left(t_{j}\right) \rightarrow 0$, we may also assume that $D_{j+1} \subset D_{j}$. Let $A_{j}$ be the spherical ring $B^{n}\left(r_{1}\right) \backslash \bar{B}^{n}\left(r_{j}\right)$. Since $g$ is injective in a neighborhood of $\partial D_{1}$, there is a component $A_{j}^{*}$ of $g^{-1} A_{j}$ such that $\partial A_{j}^{*} \supset \partial D_{1}$. Since $\partial D_{j} \cap A_{j}^{*}=\varnothing$ and since $\bar{A}_{j} \cap g B_{g}=$ $\varnothing, \bar{A}_{j}^{*} \subset U_{0} \backslash B_{g}$. By 2.2, $f$ maps $A_{j}^{*}$ homeomorphically onto $A_{j}$. Hence there is a section $s_{j}: A_{j} \rightarrow A_{j}^{*}$ of $f$. Moreover, $s_{j}=s_{k} \mid A_{j}$ for 
$k>j$. Hence we obtain a section $s: B^{n}\left(r_{1}\right) \backslash\{0\} \rightarrow U_{0} \backslash B_{g}$. By 3.10, we can extend $s$ to a continuous mapping $\bar{s}$ of $B^{n}\left(r_{1}\right)$. This is possible only if $\bar{s}(0)=0$, which implies that 0 is a removable singularity of $f$. We remark that hitherto our proof is not essentially different form that in Agard-Marden [1].

Case 2. $1 \notin \bar{T}_{0}$. We can find a path $\bar{\alpha}:[-1,1) \rightarrow R^{n}$ such that $\bar{\alpha}(-1) \in \partial U_{0}$, $\alpha(-1,1) \subset U_{0}, \bar{\alpha} \mid[0,1)=\alpha$, and $\bar{\beta}(t)=f(\bar{\alpha}(t)) \neq 0$ for $t \in[-1,1)$. By assumption, there is $\delta, 0 \leqq \delta<1$, such that $[\delta, 1) \cap T_{0}=\varnothing$. We choose an increasing sequence of points $t_{j} \in T \cap[\delta, 1)$ such that (1) $t_{j} \rightarrow 1$, (2) $|\beta(t)|<r_{j}=\left|\beta\left(t_{j}\right)\right|$ for $t \in\left(t_{j}, 1\right)$, (3) $|\bar{\beta}(t)|>r_{j+1}$ for $t \in\left[-1, t_{j}\right]$. As above, we set $D_{j}=D\left(t_{j}\right)$. There are two subcases: (a) $D_{j} \subset D_{j+1}$ for all $j$, (b) $\bar{D}_{j} \cap \bar{D}_{j+1}=\varnothing$ for some $j$.

Suppose that (a) is true. For $j>1$ let $A_{j}$ be the spherical ring $B^{n}\left(r_{1}\right) \backslash \bar{B}^{n}\left(r_{j}\right)$. As in the case 1 we conclude that there is a component $A_{j}^{*}$ of $g^{-1} A_{j}$ such that $f$ maps $A_{j}^{*}$ homeomorphically onto $A_{j}$ and such that $\partial D_{1} \subset \partial A_{j}^{*}$. Since $\alpha\left(t_{1}, 1\right) \subset \mathbf{C} \bar{D}_{1}, A_{j}^{*} \subset D_{j} \backslash \bar{D}_{1}$. Proceeding as before we obtain a section $s: B^{n}\left(r_{1}\right) \backslash\{0\} \rightarrow U_{0} \backslash B_{g}$ of $f$. Now the cluster set $C(s, 0)$ is a non-degenerate continuum in $\bar{U}_{0}^{s}$. This is in contradiction with 3.10 .

Suppose next that (b) is true. We first observe that $\alpha\left(t_{j}, 1\right) \subset \mathbf{C} \bar{D}_{j}$. Set $u_{j+1}=\sup \left\{t \mid \alpha\left(t_{j}, t\right) \subset \mathbf{C} \bar{D}_{j+1}\right\}$. Choose a neighborhood $U_{j+1}$ of $\partial D_{j+1}$ such that $f \mid U_{j+1}$ is injective. Since $\beta\left(t_{j+1}, 1\right) \subset B^{n}\left(r_{j+1}\right), g\left(U_{j+1} \cap \mathbf{C} \bar{D}_{j+1}\right) \subset$ $B^{n}\left(r_{j+1}\right)$. Hence there exists $v_{1}=\max \left\{t\left|t_{j}<t<u_{j+1},\right| \beta(t) \mid=r_{j+1}\right\}$. By definition $v_{1} \in T$. Since $v_{1}>\delta$ and since $\alpha\left(t_{j+1}, 1\right) \cap g^{-1} S^{n-1}\left(r_{j+1}\right)=$ $\varnothing=\bar{\alpha}\left(-1, t_{j}\right) \cap g^{-1} S^{n-1}\left(r_{j \div 1}\right)$, it follows that $\bar{D}\left(v_{1}\right) \subset \mathbf{C}\left(\bar{D}_{j} \cup \bar{D}_{j+1}\right)$. Hence $v_{1}^{\prime}=\sup \left\{t \mid \alpha\left(t_{j}, t\right) \subset \mathbf{C} \bar{D}\left(v_{1}\right)\right\}>t_{j}$. As above, there exists $v_{2}=\max \{t \mid$ $\left.t_{j}<t<v_{1}^{\prime},|\beta(t)|=r_{j+1}\right\}$ and we have $\bar{D}\left(v_{2}\right) \subset \mathbf{C}\left(\bar{D}_{j} \cup \bar{D}_{j+1} \cup \bar{D}\left(v_{1}\right)\right)$. By continuing this process we find an infinite number of components $\bar{C}^{*}\left(v_{i}\right)$ of $g^{-1} S^{n-1}\left(r_{j+1}\right)$ with $v_{i} \in\left(t_{j}, v_{j+1}\right)$. Hence there exists a limit point $v \in\left(t_{j}, u_{j+1}\right)$ of the set $\left\{v_{i} \mid i=1,2, \ldots\right\}$. Every neighborhood of $\alpha(v)$ intersects infinitely many components of $g^{-1} S^{n-1}\left(r_{j+1}\right)$. This is a contradiction because $f$ is a local homeomorphism at $\alpha(v)$. The theorem is proved.

3.15. Theorem. Suppose that $n \geqq 3$ and that $b$ is an essential isolated singularity of a quasimeromorphic mapping $f: G \rightarrow \bar{R}^{n}$. Then $\mathbf{C} f G \subset \overline{f B_{f}}$.

Proof. Suppose that $y \in \mathbf{C} f G \backslash \overline{f B}_{f}$. We may assume that $b=0=y$. Choose $r_{0}>0$ such that $\bar{B}^{n}\left(r_{0}\right) \subset G \cup\{0\}$ and set $U_{0}=B^{n}\left(r_{0}\right) \backslash\{0\}$. Next choose $r^{\prime}>0$ such that $\bar{B}^{n}\left(r^{\prime}\right) \cap\left(f B_{f} \cup f S^{n-1}\left(r_{0}\right)\right)=\varnothing$. By [14, 4.4], cap $\mathbf{C} f U_{0}=0$. Hence we can find a cap $C$ of $S^{n-1}\left(r^{\prime}\right)$ and a set $C^{*} \subset U_{0}$ such that $f$ maps $C^{*}$ homeomorphically onto $C$. For each $y \in C$ let $\gamma_{y}$ : $(0,1] \rightarrow R^{n}$ be the linear path $\gamma_{y}(t)=t y$. Let $\gamma_{y}^{*}$ be the (unique) maxi- 
mal lifting of $\gamma_{y}$, terminating in $C^{*}$. This means that $\gamma_{y}^{*}:\left(r_{y}, 1\right] \rightarrow U_{0}$ is a path such that $0 \leqq r_{y}<1, \gamma_{y}^{*}(1) \in C^{*}, f \circ \gamma_{y}^{*}=\gamma_{y} \mid\left(r_{y}, 1\right]$, and $\gamma_{y}^{*}(t) \rightarrow 0$ as $t \rightarrow r_{y}$. We show that $r_{y}=0$ for almost every $y \in C$. Let $E_{i}=\left\{y \in C \mid r_{y}>1 / i\right\}$. It suffices to show that $m_{n-1}\left(E_{i}\right)=0$. Set $\Gamma_{i}=$ $\left\{\gamma_{y}^{*} \mid y \in E_{i}\right\}$. Since all paths of $\Gamma_{i}$ converge to $0, M\left(\Gamma_{i}\right)=0$. By 2.14, this implies $M\left(f \Gamma_{i}\right)=0$. On the other hand, $f \Gamma_{i}$ minorizes the family 1 of all segments $\alpha_{y}:[1 / i, 1] \rightarrow R^{n}, \alpha_{y}(t)=t y, y \in E_{i}$. Consequently, a well-known formula $[26,7.7]$ yields

$$
M\left(f \Gamma_{i}\right) \geqq M(\Delta)=r^{1-n} m_{n-1}\left(E_{i}\right)(\log i)^{1-n} .
$$

Thus $m_{n-1}\left(E_{i}\right)=0$.

Choose $y \in C$ such that $r_{y}=0$. If $t \rightarrow 0$, then $\gamma_{y}^{*}(t) \rightarrow 0$ and $f\left(\gamma_{y}^{*}(t)\right)=$ $\gamma_{y}(t) \rightarrow 0$. Thus 0 is an asymptotic value of $f$ at 0 . By 3.14, this is a contradiction.

3.16. Corollary. If $n \geqq 3$ and if $f: G \rightarrow R^{n}$ is a quasiregular mapping which has an essential isolated singularity, then $f B_{f}$ is unbounded.

3.17. Corollary. If $n \geqq 3$ and if $b$ is an essential isolated singularity of a quasiregular mapping $f: G \rightarrow R^{n}$, then $b \in \bar{B}_{f}$.

3.18. Remarks. Corollary 3.17 is a special case of a theorem of Agard and Marden [1]. See also Zorič [29].

We shall indicate briefly how the proof of 3.15 can be modified so as to yield an $n$-dimensional version of Iversen's theorem: If $b$ is an essential isolated singularity of a quasimeromorphic mapping $f: G \rightarrow \bar{R}^{n}$, then every point in $\mathbf{C} f G$ is an asymptotic value of $f$. The lifting of $\gamma_{y}$ is not necessarily unique, but we can use 3.12 to find a maximal lifting $\gamma_{y}^{*}$ converging to $b$. The proof of the inequality $M\left(f \Gamma_{i}\right) \leqq K M\left(\Gamma_{i}\right)$ can be based on the idea of the proof of 2.14 together with $[13,7.10]$.

3.19. The structure of $f B_{f}$. Suppose that a quasimeromorphic mapping $f: G \rightarrow \bar{R}^{n}$ has an isolated essential singularity and that $y \in \mathbf{C} f G$. By Theorem 3.15, every neighborhood $U$ of $y$ meets $f B_{f}$. We shall now study, for $n=3$, the structure of the set $U \cap f B_{f}$. Zoric [28] has given an example of a quasiregular mapping $f: R^{3} \rightarrow R^{3}$ which has an essential singularity at $\infty$ and for which $\mathbf{C} f R^{3}=\{0, \infty\}$. In this example $f B_{f}$ consists of four rays starting from 0 . It is easy to modify this example so that $f B_{f}$ consists of three rays starting from 0 . We shall show that the number of the rays cannot be reduced to two.

We first give a factorization lemma, which is due to Church and Hemmingsen [4]. Let $(r, \varphi, z)$ be the cylindrical coordinates in $R^{n}$. Thus 
$r \geqq 0, \varphi \in R^{1}(\bmod 2 \pi), z \in R^{n-2}$, and $x_{1}=r \cos \varphi, x_{2}=r \sin \varphi,\left(x_{3}, \ldots, x_{n}\right)$ $=z$. For every non-zero integer $k$, we define the winding mapping $g_{k}: R^{n}$ $\rightarrow R^{n}$ by $g_{k}(r, \varphi, z)=(r, k \varphi, z)$. If $k>0, g_{k}$ is quasiregular with $K_{I}\left(g_{k}\right)=k, K_{o}\left(g_{k}\right)=k^{n-1}$.

3.20. Lemma. Suppose that $f: G \rightarrow R^{n}$ is discrete and open, that $x_{0} \in B_{f}$, and that $f\left(x_{0}\right)=0$. Suppose also that $x_{0}$ has a neighborhood $V$ such that $f\left(B_{f} \cap V\right)$ is contained in the (n-2)-dimensional subspace $Z=\left\{y \in R^{n} \mid y_{1}=\right.$ $\left.y_{2}=0\right\}$. Then there is a neighborhood $U=U\left(x_{0}, f, r\right)$ of $x_{0}$ and a homeomorphism $h$ of $U$ onto a ball $B^{n}(r)$ such that $f \mid U=g_{k} \circ h, k=i\left(x_{0}, f\right)$.

Proof. Choose $r>0$ such that $U=U\left(x_{0}, f, r\right)$ is a normal neighborhood of $x_{0}$ and $U \subset V$. Set $g=f: U$. Since $f$ defines a $k$-to-one covering mapping of $U \backslash g^{-1} g B_{g}$ onto $B^{n}(r) \backslash g B_{g}, B^{n}(r) \backslash g B_{g}$ is not simply connected (cf. [4, 5.2]). Since $g B_{g} \subset Z$, this implies $g B_{g}=Z \cap B^{n}(r)$. The lemma follows from $[4,4.1]$.

3.21. Definition. A set $A \subset \bar{R}^{n}$ is said to be a quasiconformal p-ball if there is a neighborhood $U$ of $A$ and a quasiconformal mapping $g$ of $U$ such that $g A=B^{p}$. If $p=1, A$ is called a quasiconformal arc.

3.22. Theorem. Suppose that $b$ is an isolated essential singularity of a quasimeromorphic mapping $f: G \rightarrow \bar{R}^{3}$ and that $y \in \mathbf{C} f G$. Then $V \cap f B_{f}$ is not contained in a quasiconformal are for any neighborhood $V$ of $y$.

Proof. The idea of the proof is much similar to that used in Zorič [28], [29], Agard-Marden [1], and in the proofs of 2.3 and 3.14. We shall therefore omit some details. We may assume that $b=0=y$. Suppose that $V \cap f B_{f}$ is contained in a quasiconformal are for some neighborhood $V$ of 0 . We may assume that $V \cap f B_{f} \subset Z=\left\{x \in R^{3} \mid x_{1}=x_{2}=0\right\}$. Fix $r_{0}>0$ such that $\bar{B}^{3}\left(r_{0}\right) \subset G \cup\{0\}$, and set $U_{0}=B^{3}\left(r_{0}\right) \backslash\{0\}, g=$ $f \mid U_{0}$. By 3.15, there is $r^{\prime} \neq 0$ such that $r^{\prime} e_{3} \in g B_{g}, \bar{B}^{3}\left(\left|r^{\prime}\right|\right) \subset V$ and $\bar{B}^{3}\left(\left|r^{\prime}\right|\right) \cap f S^{2}\left(r_{0}\right)=\varnothing$. We may assume that $r^{\prime}>0$. Choose $x_{0} \in g^{-1}\left(r^{\prime} e_{3}\right)$ $\cap B_{g}$. By 3.20, $g$ is topologically equivalent to a winding mapping $g_{k}$ in a neighborhood of $x_{0}$. Let $\beta:\left(0, r^{\prime}\right] \rightarrow R^{3}$ be the path $\beta(t)=t e_{3}$. Using 3.12 we choose a maximal lifting $\alpha:\left(\delta, r^{\prime}\right] \rightarrow G$ of $\beta$ terminating at $x_{0}$. From 3.20 it follows that for every $t \in\left(\delta, r^{\prime}\right] g$ is topologically equivalent to $g_{k}$ in some neighborhood of $\alpha(t)$. Furthermore, it follows from 3.12 that $\alpha(t) \rightarrow \partial U_{0}$ as $t \rightarrow \delta$, which is possible only if $\alpha(t) \rightarrow 0$.

For $0<r<r^{\prime}-\delta$ consider the cap

$$
C(r, \varphi)=\left\{y \in R^{3}|| y-\delta e_{3} \mid=r, y_{3}>\delta+r \cos \varphi\right\},
$$


$0<\varphi \leqq \pi$. Let $C^{*}(r, \varphi)$ be the component of $g^{-1} C(r, \varphi)$ which contains $\alpha(\delta+r)$. Let $\varphi_{r}$ be the least upper bound of all $\varphi \in(0, \pi]$ such that $C^{*}(r, \varphi)$ is homeomorphic to $C(r, \varphi)$, and such that $f$ defines a $k$-to-one covering mapping of $C^{*}(r, \varphi) \backslash|\alpha|$ onto $C(r, \varphi) \backslash|\beta|$. Let $E=$ $\left\{r \in\left(0, r^{\prime}-\delta\right) \mid 0 \in \bar{C}^{*}\left(r, \varphi_{r}\right)\right\}$. Using similar path family estimates as in the proof of 3.14 and applying 2.14 we see that $m_{1}(E)=0$. It is not difficult to see that if $r \in\left(0, r^{\prime}-\delta\right) \backslash E$, then $\varphi_{r}=\pi, \bar{C}^{*}\left(r, \varphi_{r}\right)$ is a topological 2-sphere, and the mapping $\bar{C} *\left(r, \varphi_{r}\right) \rightarrow \bar{C}\left(r, \varphi_{r}\right)=S^{2}\left(\delta e_{3}, r\right)$ is topologically equivalent to $g_{k} \mid S^{2}$. Choose a sequence $r_{1}>r_{2}>\ldots$ such that $r_{i} \in\left(0, r^{\prime}-\delta\right) \backslash E$ and $r_{i} \rightarrow 0$. If $\delta>0$. we choose $r_{1}<\delta$. Let $D_{i}$ be the bounded component of $\mathbf{C} \bar{C}^{*}\left(r_{i}, \varphi_{r_{i}}\right)$. Then $\bar{D}_{i} \subset B^{3}\left(r_{0}\right)$, and each $\partial D_{i}$ separates the points $x_{0}$ and 0 . Passing to a subsequence we may assume that one of the following cases occurs: (1) $0 \in D_{i+1} \subset D_{i}$ for all $i$ or (2) $x_{0} \in D_{i} \subset D_{i+1}$ for all $i$.

Suppose that (1) is true. We first show that $A_{i}=D_{i} \backslash \bar{D}_{i+1}$ is a normal domain. Suppose that this is not true. Then $A_{i} \cap f^{-1} f \partial A_{i} \neq \varnothing$. Let $Q$ be a component of this set. From 3.7 it follows that there is a neighborhood $U$ of $\partial A_{i}$ such that $M^{*}(f, U)=k$. Hence $U \cap Q=\varnothing$, which implies that $Q$ is compact. From [27, (7.5), p. 148] it follows that $f Q=S_{j}=$ $S^{2}\left(\delta e_{3}, r_{j}\right)$ where $j=i$ or $i+1$. Suppose first that $\delta=0$. Let $\beta_{j}:\left(0, r_{j}\right]$ $\rightarrow R^{3}$ be the path $\beta_{j}(t)=t e_{3}$. By $3.12, \beta_{j}$ has a maximal lifting $\alpha_{j}:\left(c_{j}, r_{j}\right]$ $\rightarrow G$ terminating at a point $x_{1} \in Q$. Moreover, $\alpha_{j}(t) \rightarrow 0$ as $t \rightarrow c_{j}$. Hence there is $t_{0} \in\left(c_{j}, r_{j}\right)$ such that $\alpha_{j}\left(t_{0}\right) \in \partial D_{i+1}$. If $j=i+1$, this is clearly impossible. If $j=i$, then $t_{0}=r_{i+1}$, and $\alpha_{i}\left(t_{0}\right)$ is the unique point in $\partial D_{i+1} \cap f^{-1}\left(r_{i+1} e_{3}\right)$. Hence $\alpha_{i} \mid\left[r_{i+1}, r_{i}\right]$ and $\alpha\left[\left[r_{i+1}, r_{i}\right]\right.$ are both liftings of $\beta \mid\left[r_{i+1}, r_{i}\right]$ starting at $\alpha\left(r_{i+1}\right)$. Since $i(\alpha(t), f)$ is constant, it follows from the last statement of 3.12 that $\alpha(t)=\alpha_{i}(t)$ for $t \in\left[r_{i+1}, r_{i}\right]$, which is impossible. Next assume that $\delta>0$. We let $\beta_{j}^{\prime}:\left(0, \delta-r_{j}\right] \rightarrow R^{3}$ be the path $\beta_{j}^{\prime}(t)=t e_{3}$. Choose a maximal lifting $\alpha_{j}^{\prime}:\left(c_{j}^{\prime}, \delta-r_{j}\right] \rightarrow G$ of $\beta_{j}^{\prime}$ terminating at a point in $Q$. Then $\alpha_{j}^{\prime}(t) \rightarrow 0$ as $t \rightarrow c_{j}^{\prime}$. Hence there is $t_{0}^{\prime} \in\left(c_{j}^{\prime}\right.$, $\left.\delta-r_{j}\right)$ such that $\alpha_{j}^{\prime}\left(t_{0}^{\prime}\right) \in \partial D_{j+1}$. This is clearly impossible in both cases $j=i$ ard $j=i+1$. We have proved that $A_{i}$ is a normal domain.

Let $\Gamma_{i}$ be the family of all paths joining the boundary components of $A_{i}$ in $A_{i}$. Lemma 3.9 yields $N\left(f, A_{i}\right)=N\left(f, \partial A_{i}\right)=k$. Using $[13,3.2$ and 5.9] we obtain

$$
\left(b_{3} \frac{d\left(D_{i}\right)^{3}}{m\left(D_{1}\right)}\right)^{\frac{1}{2}} \leqq M\left(\Gamma_{i}\right) \leqq k K(f) M\left(f \Gamma_{i}\right) \leqq 4 \pi k K(f)\left(\log \frac{r_{1}}{r_{i}}\right)^{-2}
$$

Hence $d\left(D_{i}\right) \rightarrow 0$ as $i \rightarrow \infty$. Thus $f\left(D_{1} \backslash\{0\}\right) \subset B^{3}\left(r_{1}\right)$, which is a contradiction by $[14,4.6]$.

Suppose next that (2) is true. As above, we see that the $\operatorname{ring} A_{i}=$ 
$D_{i} \backslash \bar{D}_{1}$ is a normal domain. Using similar path family estimates we obtain

$$
\left(b_{3} \frac{m\left(D_{1}\right)^{3}}{m\left(U_{0}\right)}\right)^{\frac{1}{2}} \leqq M\left(\Gamma_{i}\right) \leqq k K(f) M\left(f \Gamma_{i}\right) \leqq 4 \pi k K(f)\left(\log \frac{r_{1}}{r_{i}}\right)^{-2} .
$$

As $i \rightarrow \infty$, this gives a contradiction. The theorem is proved.

3.23. Remark. The function $f(z)=e^{z}$ shows that none of the results $3.14,3.15,3.16,3.17,3.22$ is true for $n=2$.

3.24. Tamely embedded branch set image. Now we shall study the case where $f B_{f}$ is a tamely embedded $(n-2)$-manifold. From the theorem of Church and Hemmingsen (Lemma 3.20 above) it follows that $B_{f}$ is also tamely embedded and that $f$ is locally topologically equivalent to a winding mapping $g_{k}$. If $f$ is quasiregular, we can go a bit further.

3.25. Theorem. Let $f: G \rightarrow R^{n}$ be quasiregular and let $x_{0} \in B_{f}$. Suppose that there is a neighborhood $U$ of $x_{0}$ such that $f\left(U \cap B_{f}\right)$ is contained in a quasiconformal (n-2)-ball. Then there exists a neighborhood $V$ of $x_{0}$ and quasiconformal mappings $h_{1}: V \rightarrow B^{n}, h_{2}: B^{n} \rightarrow V^{\prime} \subset f U$ such that $f \mid V=h_{2} \circ g_{k} \circ h_{1}$, where $k=i\left(x_{0}, f\right)$. In particular, $B_{f} \cap V$ is a quasiconformal $(n-2)$-ball.

Proof. There is a neighborhood $V^{\prime}$ of $f\left(x_{0}\right)$ and a quasiconformal mapping $g: V^{\prime} \rightarrow B^{n}$ such that $V^{\prime} \subset f U, g\left(f\left(x_{0}\right)\right)=0$, and $g f\left(U \cap B_{f}\right)$ $\subset Z=\left\{x \in R^{n} \mid x_{1}=x_{2}=0\right\}$. By 3.20, there is a neighborhood $V$ of $x_{0}$ and a homeomorphism $k_{1}: V \rightarrow B^{n}(r)$ such that $g \circ f: V=g_{k} \circ k_{1}$. Setting $h_{1}(x)=k_{1}(x) / r$ and $h_{2}(y)=g^{-1}(r y)$ we have $f \mid V=h_{2} \circ g_{k} \circ h_{1}$. It remains to show that $h_{1}$ is quasiconformal. Every point $w \in B^{n} \backslash Z$ has a neighborhood $W$ such that $\varphi=f \mid h_{1}^{-1} W$ is injective. Since $h_{1}^{-1}\left|W=\varphi^{-1} \circ h_{2} \circ g_{k}\right|$ $W, h_{1}^{-1}$ is locally $\mathrm{K}$-quasiconformal in $B^{n} \backslash Z$, where $K=K(f) K\left(h_{2}\right)$ $K\left(g_{k}\right)$. Since $\Lambda_{n-1}(Z)=0$, it follows from $[6, p .380]$ or $[26,35.1]$ that $h_{1}^{-1}$, and hence $h_{1}$ is quasiconformal.

3.26. We shall next study the case $n=3$ in more detail. A bounded arc $J \subset R^{n}$ is said to have bounded oscillation [11, p. 107] if there is a constant $a<\infty$ such that $\left|x_{1}-x_{2}\right| \leqq a\left|x_{1}-x_{3}\right|$ holds for successive points $x_{1}, x_{2}, x_{3}$ of $J$. We shall show that if $f B_{f}$ is a tamely embedded arc, then both $B_{f}$ and $f B_{f}$ are ares with locally bounded oscillation. In particular, if they are piecewise smooth arcs, no zero angles can occur. For example, $f B_{f}$ cannot be the set $\left\{x \in R^{3}\left|x_{3}=0, x_{1} \geqq 0,\right| x_{2} \mid=x_{1}^{2}\right\}$. We first need a modulus estimate. 
3.27. Lemma. Suppose that $F \subset \bar{R}^{n}$ is connected and that $\alpha:[0,1] \rightarrow \mathbf{C} F$ is a loop which is not homotopically trivial in $\mathbf{C} F$. Let $\Gamma$ be the family of all paths which join $|x|$ and $F$. Then $M(\Gamma) \geqq c_{n}>0$ where $c_{n}$ depends only on $n$.

Proof. Choose $a \in \mid \wedge$ and $b \in \bar{F}$ such that $|a-b|=d(|\alpha|, F)=s$. If $s=0$, the spheres $S^{n-1}(a, t)$ meet both $|\alpha|$ and $F$ for sufficiently small $t$, and $M(\Gamma)=\infty$ by [26, 10.12]. If $s>0$, we set $x_{0}=(a+b) / 2$. Since $\alpha$ is not homotopically trivial in $\mathbf{C} F,|\alpha|$ contains a point in $S^{n-1}(a, s) \backslash B^{n}(b, s)$. Since $\mathbf{C} B^{n}(b, s)$ is simply connected, $F$ contains a point in $S^{n-1}(b, s) \backslash B^{n}(a, s)$. Hence the sphere $S^{n-1}\left(x_{0}, t\right)$ meets both $|\alpha|$ and $F$ whenever $s / 2<t<s \sqrt{3} / 2$. Hence [26, 10.12] yields $M(\Gamma) \geqq b_{n} \log \sqrt{3}$.

3.28. The linking number. We also need the notion of a linking number, see e.g. Spanier [22, p. 361]. If the orientation of $\bar{R}^{3}$ is fixed, the linking number $\operatorname{lk}(y, z)$ is an integer, defined for 1-dimensional singular cycles $y, z$ in $\bar{R}^{3}$ such that $y \cap z:=\varnothing$. We recall some properties of the linking number.

(1) $\operatorname{lk}(y, z)=\operatorname{lk}(z, y)$.

(2) $\operatorname{lk}(y, z)+\operatorname{lk}\left(y^{\prime}, z\right)=\operatorname{lk}\left(y+y^{\prime}, z\right)$.

(3) If $y \sim y^{\prime}$ in $\mathbf{C}|z|$, then $\operatorname{lk}(y, z)=\operatorname{lk}\left(y^{\prime}, z\right)$. In particular, $\operatorname{lk}(y, z)=0$ if $y$ bounds in $\mathbf{C}|z|$.

(4) If $y$ represents a line in $\bar{R}^{3}$ and if $z$ represents an orthogonal circle with center in $|y|$, then $\operatorname{lk}(y, z)= \pm \mathbf{1}$.

(5) The linking number is invariant under sense-preserving homeomorphisms.

3.29. Lemma. Let $k \geqq 2$, let $g_{k}: R^{3} \rightarrow R^{3}$ be the winding mapping $g_{k}(r, \varphi, z)=(r, k \varphi, z)$, and let $Z$ be the line $\left\{t e_{3}: t \in R^{1}\right\}$. Suppose that $-1<a<b<c<1$, and that $E, F$ are open arcs such that $E$ joins be $e_{3}$ and a point $P \in S^{2} \backslash Z$ in $B^{3} \backslash Z$, and such that $F$ joins ae $e_{3}$ and $c e_{3}$ in $B^{3} \backslash Z \backslash E$. Then $g_{k}^{-1} \bar{F}$ contains a loop which is not homotopically trivial in $B^{3} \backslash g_{k}^{-1} \bar{E}$.

Proof. Let $h: \bar{R}^{3} \rightarrow \bar{R}^{3}$ be the rotation $h(r, \varphi, z)=(r, \varphi+2 \pi / k, z)$. Fix a component $E_{0}$ of $g_{k}^{-1} E$ and a component $F_{0}$ of $g_{k}^{-1} F$. Let $\left(r_{0}, \varphi_{0}, z_{0}\right)$ be the unique point in $\bar{E}_{0} \cap S^{2}$. We choose injective singular 1-simplexes $\sigma_{0}, \tau_{0}, \sigma, \tau$ such that $\left|\sigma_{0}\right|=\bar{E}_{0},\left|\tau_{0}\right|=\bar{F}_{0},|\sigma|=\left\{\left(r_{0}, \varphi, z_{0}\right) \mid \varphi_{0} \leqq \varphi \leqq\right.$ $\left.\varphi_{0}+2 \pi \mid k\right\},|\tau|=\left(Z \backslash\left\{t e_{3} \mid a<t<c\right\}\right) \cup\{\infty\}$, and choose the orientations so that the chains $y_{0}=\sigma_{0}+\sigma-h \sigma_{0}$ and $w_{0}=\tau_{0}+\tau$ are cycles. 
For $0 \leqq j \leqq k-1$ we define the cycles $y_{j}=h y_{j-1}$ and $w_{j}=h w_{j-1}$. Then $y=\Sigma y_{j}$ represents the circle $r=r_{0}, z=z_{0}$. Since $w_{0}$ is in the obvious sense homotopic to $Z \cup\{\infty\}$ in $\mathbf{C}|y|$, it follows from (3) and (4) in 3.28 that $\operatorname{lk}\left(y, w_{0}\right)= \pm 1$. On the other hand, 3.28 (5) implies $\operatorname{lk}\left(y_{0}, w_{j}\right)=\operatorname{lk}\left(y_{k-j}, w_{0}\right)$ for $1 \leqq j \leqq k-1$. Hence

$$
\sum_{j=0}^{k-1} 1 \mathrm{k}\left(y_{0}, w_{j}\right)=\sum_{j=0}^{k-1} \operatorname{lk}\left(y_{j}, w_{0}\right)=\operatorname{lk}\left(y, w_{0}\right)= \pm 1 .
$$

This implies

$$
\sum_{j=0}^{k-1} \operatorname{lk}\left(y_{0}, w_{j}-w_{0}\right)= \pm 1-k \operatorname{lk}\left(y_{0}, w_{0}\right) \neq 0 .
$$

Hence there is $j$ such that $\operatorname{lk}\left(y_{0}, w_{j}-w_{0}\right) \neq 0$. On the other hand, $w_{j}-w_{0}=$ $h^{j} \tau_{0}-\tau_{0}$ represents a Jordan curve $J \subset g_{k}^{-1} \bar{F}$. By $3.28(3), w_{j}-w_{0} \sim 0$ in $\mathbf{C}\left|y_{0}\right| \supset B^{3} \backslash g_{k}^{-1} \bar{E}$. Hence $J$ is not homotopically trivial in $B^{3} \backslash g_{k}^{-1} \bar{E}$.

3.30. Theorem. Let $f: G \rightarrow R^{3}$ be a quasiregular mapping, let $x \in B_{f}$, and suppose that there exist a neighborhood $U$ of $x$ and a homeomorphism $g: f U \rightarrow B^{3}$ such that $g(f(x))=0$ and $g f\left(B_{f} \cap U\right)=B^{1}$. Then there is a neighborhood $V$ of $x$ such that $V \cap B_{f}$ and $f\left(V \cap B_{f}\right)$ are Jordan arcs with bounded oscillation.

Proof. We first show that $f\left(W \cap B_{f}\right)$ has bounded oscillation for some neighborhood $W$ of $x$. By 3.20, there exist $r_{1}>0$ and a homeomorphism $h$ of $\bar{U}_{1}=\bar{U}\left(x, g \circ f, r_{1}\right)$ onto $\tilde{E}^{3}\left(r_{1}\right)$ such that $g \circ f \mid \bar{U}_{1}=g_{k} \circ h$, where $k=i(x, f)$. Choose $t>0$ such that $B^{3}(f(x), 2 t) \subset f L_{1}$. Next choose $r>0$ such that $U(f(x), g, r) \subset B^{3}(f(x), t)$. Set $W=U(x, g \circ f, r)$ and $J=B_{f} \cap W$. We claim that $J^{\prime}=f J$ has bounded oscillation. Suppose that this is not the case. Then there is for every $m>1$ a triple $y_{1}, y_{2}, y_{3}$ of successive points of $J^{\prime}$ such that $\left|y_{1}-y_{2}\right|>m\left|y_{1}-y_{3}\right|$. Choose an open arc $E \subset f\left(U_{1} \backslash B_{f}\right) \backslash B^{3}\left(y_{1}, \mid y_{1}-y_{2}\right)$ which joins $y_{2}$ and a point $P \in \partial f U_{1}$. Next choose an open arc $F \subset B^{3}(f(x), t) \cap B^{3}\left(y_{1},\left|y_{1}-y_{3}\right|\right)$ $\backslash f\left(B_{f} \cap U_{1}\right)$ which joins $y_{1}$ and $y_{3}$. Let $\Gamma$ be the family of all paths joining $U_{1} \cap f^{-1} \bar{F}$ and $\partial U_{1} \cup f^{-1} \bar{E}$ in $U_{1}$. Since $f \mid \bar{U}_{1}$ is topologically equivalent to $g_{k}$, it follows from Lemma 3.29 that there is a loop $\alpha$ in $U_{1} \cap f^{-1} \bar{F}$ which is not homotopically trivial in $U_{1} \backslash f^{-1} \bar{E}$. Since $\Gamma$ minorizes the family of all paths joining $|\alpha|$ and $\partial U_{1} \cup\left(U_{1} \cap f^{-1} \bar{E}\right)$, 3.27 implies $M(\Gamma) \geqq c_{3}>0$. On the other hand, $M(f \Gamma) \leqq 4 \pi /(\log m)^{2}$. By [13, 3.2], we have $M(\Gamma) \leqq k K_{o}(f) M(f \Gamma)$. Thus $c_{3}(\log m)^{2} \leqq 4 \pi k K_{o}(f)$. Letting $m \rightarrow \infty$ yields a contradiction. Thus $J^{\prime}$ has bounded oscillation.

Choose now $s>0$ such that $V=U(x, g \circ f, s) \subset B^{3}(x, u)$ and $B^{3}(x, 2 u) \subset W$ for some $u$. We claim that the arc $J_{0}=B_{f} \cap V$ has 
bounded oscillation. If not, there is for every $m>1$ a triple $x_{1}, x_{2}, x_{3}$ of successive points of $J_{0}$ such that $\left|x_{1}-x_{2}\right|>m\left|x_{1}-x_{3}\right|$. We may assume that $\left|f\left(x_{1}\right)-f\left(x_{2}\right)\right| \leqq\left|f\left(x_{2}\right)-f\left(x_{3}\right)\right|$. Let $x_{0}$ be the end point of $J$ such that $x_{0}, x_{3}, x_{2}$ are successive points of $\bar{J}$. Let $\alpha_{1}$ be the open subarc of $J_{0}$ with end points $x_{1}$ and $x_{2}$, and let $\alpha_{2}$ be the open subarc of $J$ with end points $x_{0}$ and $x_{3}$. Let $\Gamma$ be the family of all paths which join $\alpha_{1}$ and $\alpha_{2}$ in $W$. If $\left|x_{1}-x_{3}\right|<t<\left|x_{1}-x_{2}\right|$, the sphere $S^{2}\left(x_{1}, t\right)$ meets both $\alpha_{1}$ and $\alpha_{2}$. Hence $[26,10.12]$ implies

$$
M(\Gamma) \geqq b_{3} \log \frac{\left|x_{1}-x_{2}\right|}{\left|x_{1}-x_{3}\right|} \geqq b_{3} \log m .
$$

We next derive an upper bound for $M(f \Gamma)$. By the first part of the proof, there exists a constant $a$ such that $\left|z_{1}-z_{2}\right| \leqq a\left|z_{1}-z_{3}\right|$ for successive points $z_{1}, z_{2}, z_{3}$ of $J^{\prime}$. Set $\beta_{i}=f \alpha_{i}$, and suppose $y_{i} \in \beta_{i}, i=1,2$. Then $\delta=\left|f\left(x_{1}\right)-f\left(x_{2}\right)\right| \leqq\left|f\left(x_{2}\right)-f\left(x_{3}\right)\right| \leqq\left|f\left(x_{2}\right)-y_{1}\right|+\left|f\left(x_{3}\right)-y_{1}\right| \leqq 2 a\left|y_{1}-y_{2}\right|$. Hence $d\left(\beta_{1}, \beta_{2}\right) \geqq \delta / 2 a$. Define $\varrho: R^{3} \rightarrow R^{1}$ by setting $\varrho(z)=2 a / \delta$ for $z \in \beta_{1}+B^{3}(\delta / 2 a)$ and $\varrho(z)=0$ elsewhere. Then $\varrho \in F(f I)$. Since $\left|y_{1}-f\left(x_{1}\right)\right|$ $\leqq a \delta, \varrho(z)=0$ whenever $\left|z-f\left(x_{1}\right)\right| \geqq a \delta+\delta / 2 a$. Hence

$$
M(f \Gamma) \leqq \int \varrho^{3} d m \leqq 8 \Omega_{3} a^{3}(a+1 / 2 a)^{3} .
$$

By $[13,3.2]$, we again have $M(\Gamma) \leqq k K_{0}(f) M(f \Gamma)$, which gives the contradiction as $m \rightarrow \infty$. The theorem is proved.

3.31. Remark. If a planar arc has bounded oscillation, it is a quasiconformal are (Rickman [20], cf. also [11, p. 104]). The corresponding result in $R^{3}$ is not true, because there exist wild arcs with bounded oscillation. The authors do not know whether a tame are with bounded oscillation must be quasiconformal.

\section{Bounds for the local degree}

4.1. It has been conjectured that for $n \geqq 3$ the local degree $i(x, f)$ is bounded by a constant which depends only on $n$ and $K(f)$. This was disproved by Rickman [21] who constructed quasiregular mappings $f: R^{3} \rightarrow R^{3}$ such that $i(0, f)$ was arbitrarily large while $K(f)$ was bounded by a universal constant. In this example, whose $n$-dimensional version will be given in $4.9, B_{f}$ consisted of a family of rays starting from the origin, and the local degree on $B_{f}$ was 2,3 , or 4 , except at the origin. In this section we show that this example is typical in two respects: (1) $i(x, f)$ cannot be large at every point of $B_{f}$. (2) If $i\left(x_{0}, f\right)$ is large, then $B_{f}$ 
must lie on »every side» of $x_{0}$. We also show that a quasiregular mapping is a local homeomorphism or constant if its dilatation is sufficiently close to 1 . We close with some counterexamples.

4.2. Theorem. Let $n \geqq 3$ and let $f: G \rightarrow R^{n}$ be a non-constant quasiregular mapping. Then either $B_{f}=\varnothing$ or

$$
\inf _{x \in B_{f}} i(x, f)<\left(\frac{n}{n-2}\right)^{n-1} K_{I}(f) \leqq 9 K_{I}(f) .
$$

Proof. Since $A_{n-2}\left(f B_{f}\right)>0$ by 3.4 , the theorem follows directly from Martio [12, 6.8].

4.3. Lemma. Let $f: G \rightarrow R^{n}$ be a non-constant quasiregular mapping and let $x_{0} \in G$. Then there are $r>0$ and $C>0$ such that for $x \in B^{n}\left(x_{0}, r\right)$

$$
\left|f(x)-f\left(x_{0}\right)\right| \leqq C\left|x-x_{0}\right|^{\prime \prime}
$$

where $\mu=\left(i\left(x_{0}, f\right) / K_{I}(f)\right)^{1 /(n-1)}$.

Proof. This was proved in Martio [12, 6.1] although the formulation was less general.

4.4. Theorem. Suppose that $n \geqq 3$, that $f: G \rightarrow R^{n}$ is $K$-quasiregular, and that $\Delta$ is an open cone in $G \backslash B_{f}$ with vertex at $x_{0} \in G$ and angle $\alpha$. Then $i\left(x_{0}, f\right) \leqq C$ where $C$ depends on $n, K$, and $\alpha$.

Proof. Performing auxiliary similarity mappings we may assume, in view of Lemma 4.3, that (1) $x_{0}=0=f\left(x_{0}\right),(2) d=\left\{x|| x \mid \cos \alpha<x_{1}<1\right\}$, (3) if $x \in \Delta$, then $|f(x)| \leqq|x|^{\mu}$ where $\mu^{n-1}=i\left(x_{0}, f\right) / K$. By 2.7, $f$ is injective in the ball $B_{t}=B^{n}\left(t e_{1}, t \psi(n, K) \sin \alpha\right), 0<t \leqq 1 / 2$. Let $J_{t}$ be the segment $\left\{s e_{1} \mid a t \leqq s \leqq t\right\}$ where $a=1-\frac{1}{2} \psi(n, K) \sin \alpha$. Then $E_{t}=\left(B_{t}, J_{t}\right)$ is a condenser, and cap $E_{t}$ depends only on $n$.

Pick $t_{0} \in(0,1 / 2]$ such that $f^{-1}(0) \cap B^{n}\left(2 t_{0}\right)=\{0\}$. If $0<t \leqq t_{0}$, then $f E_{t}$ is a rirglike condenser, and $\mathbf{C} f B_{t}$ contains 0 and $\infty$. Setting $g(t)=$ $\left|f\left(t e_{1}\right)\right|$ and using a well-known modulus estimate $[26,10.12]$ yield

$$
\operatorname{cap} f E_{t} \geqq b_{n} \log \frac{g(t)}{g(a t)}
$$

where $b_{n}>0$ depends only on $n$. Since $K$ cap $E_{t} \geqq \operatorname{cap} f E_{t}$, we obtain an inequality

$$
\frac{g(t)}{g(a t)} \leqq C_{0}
$$


where $C_{0}$ depends on $n$ and $K$. Since this holds for all $t \in\left(0, t_{0}\right]$, we obtain by iteration

$$
g\left(t_{0}\right) \leqq C_{0} g\left(a t_{0}\right) \leqq C_{0}^{2} g\left(a^{2} t_{0}\right) \leqq \ldots \leqq C_{0}^{k} g\left(a^{k} t_{0}\right) .
$$

On the other hand, $g(t) \leqq t^{\mu}$, which implies $g\left(t_{0}\right) \leqq C_{0}^{k} a^{k \mu} t_{0}^{\mu}$. Letting $k \rightarrow \infty$ yields $C_{0} a^{\mu} \geqq 1$. Thus

$$
i\left(x_{0}, f\right) / K=\mu^{n-1} \leqq\left(\frac{\log C_{0}}{\log (1 / a)}\right)^{n-1} .
$$

The theorem is proved.

4.5. Lemma. Suppose that $f_{j}: G \rightarrow R^{n}$ is a sequence of sense-preserving discrete open mappings which converge to a discrete open mapping $f: G \rightarrow R^{n}$ uniformly on every compact part of $G$. Then $i\left(x_{0}, f\right) \geqq \lim \sup i\left(x_{0}, f_{j}\right)$ for every $x_{0} \in G$.

Proof. Choose $r>0$ such that $D=U\left(x_{0}, f, r\right)$ is a normal neighborhood of $x_{0}$ with respect to $f$. Then $i\left(x_{0}, f\right)=\mu\left(y_{0}, f, D\right)$, where $y_{0}=f\left(x_{0}\right)$. Since $f_{j} \rightarrow f$ uniformly on $\bar{D}$, there is $j_{0}$ such that $\left|f_{j}(x)-f(x)\right|$ $<r / 2$ for $j \geqq j_{0}$ and $x \in \bar{D}$. By [16, Theorem 6, p. 131] this implies $\mu\left(f_{j}\left(x_{0}\right), f_{j}, D\right)=\mu\left(y_{0}, f, D\right)=i\left(x_{0}, f\right)$ for $j \geqq j_{0}$. On the other hand, $\mu\left(f_{j}\left(x_{0}\right), f_{j}, D\right)=\Sigma i\left(x, f_{j}\right)$ over $x \in D \cap f_{j}^{-1}\left(f_{j}\left(x_{0}\right)\right)$. Thus $\mu\left(f_{j}\left(x_{0}\right), f_{j}, D\right)$ $\geqq i\left(x_{0}, f_{j}\right)$, and the lemma follows.

4.6. Theorem. For every $n \geqq 3$ there is $K>1$ such that every nonconstant K-quasiregular mapping $f: G \rightarrow R^{n}$ is a local homeomorphism.

Proof. Suppose that the theorem is not true for some $n \geqq 3$. Then there exists a sequence of non-constant $K_{j}$-quasiregular mappings $f_{j}: G_{j} \rightarrow R^{n}$ such that $K_{j} \rightarrow 1$ and $B_{f_{j}} \neq \varnothing$ for all $j$. We may assume that $K_{j} \leqq 2$ for all $j$. By 4.2, there is $x_{j} \in B_{f_{j}}$ such that $i\left(x_{j}, f_{j}\right) \leqq 18$. From $[13,4.5]$ it follows that $H\left(x_{j}, f_{j}\right)<C$ where $C$ depends only on $n$. By $[13,2.9]$, $U\left(x_{j}, f_{j}, r\right)$ is a normal neighborhood of $x_{j}$ for sufficiently small $r$. Performing auxiliary similarity mappings we may assume that (i) $x_{j}=$ $0=f\left(x_{j}\right)$, (ii) $U\left(0, f_{j}, r\right)$ is a normal neighborhood of 0 for $0<r \leqq 1$, (iii) $l *\left(0, f_{j}, 1\right)=1$, (iv) $L\left(0, f_{j}, t\right)<C l\left(0, f_{j}, t\right)$ for $0<t \leqq 1$. Observe that (iii) implies $\bar{B}^{n} \subset G_{j}$.

Since $f_{i} B^{n} \subset B^{n}$, it follows from $[14,3.17]$ that the restrictions $g_{j}=$ $f_{j} \mid B^{n}$ form a normal family. Passing to a subsequence, we may therefore assume that $g_{j} \rightarrow g$ uniformly on every compact part of $B^{n}$. By Rešetnjak [19, p. 664], $g: B^{n} \rightarrow R^{n}$ is 1-quasiregular. A 1-quasiregular mapping is either constant or a Möbius transformation. This was proved by Rešetnjak 
in [18], but it follows also easily from the corresponding result for quasiconformal mappings (Gehring [6, p. 388], Rešetnjak [17]) and from the condition $\operatorname{dim} B_{f} \leqq n-2$. Since $0 \in B_{f_{j}}$, it follows from 4.5 that $g$ cannot be a homeomorphism. Hence $g$ is constant. Since $g_{j}(0)=0, g(x)=0$ for all $x \in B^{n}$.

Set $l_{j}=l\left(0, f_{j}, 1\right)$. Since $L\left(0, f_{j}, 1\right)=1$, (iv) implies $l_{j} \geqq 1 / C$. Let $E_{j}$ be the condenser $\left(U\left(0, f_{j}, l_{j}\right), \bar{U}\left(0, f_{j}, l_{j} / 2\right)\right)$. Then $f_{j} E_{j}=$ $\left(B^{n}\left(l_{j}\right), \bar{B}^{n}\left(l_{j} / 2\right)\right)$, and cap $f_{j} E_{j}=\omega_{n-1}(\log 2)^{1-n}$. Since $L^{*}\left(0, f_{j}, l_{j}\right)=1$, we obtain the estimate $\operatorname{cap} E_{j} \geqq \omega_{n-1}\left(\log \left(1 / l_{j}^{*}\right)\right)^{1-n} \quad$ where $l_{j}^{*}=$ $l_{j}^{*}\left(0, f_{j}, l_{j} / 2\right)$. Since $E_{i}$ is a normal condenser of $f_{j}$, we have by $[13,6.2]$

$$
\operatorname{cap} E_{j} \leqq K_{j} N\left(f_{j}, E_{j}\right) \operatorname{cap} f E_{j} \leqq 36 \omega_{n-1}(\log 2)^{1-n} .
$$

Combining the above inequalities yields $l_{j}^{*} \leqq C_{1}<1$ where $C_{1}$ depends only on $n$. For each $j$ there is $a_{j} \in \partial U\left(0, f_{j}, l_{j} / 2\right)$ such that $\left|a_{j}\right|=l_{j}^{*}$. Then $\left|g_{j}\left(a_{j}\right)\right|=l_{j} / 2 \geqq 1 / 2 C$, which contradicts the fact that $g_{j} \rightarrow 0$ uniformly on $\bar{B}^{n}\left(C_{1}\right)$. The theorem is proved.

4.7. Remark. Theorem 4.6 does not give any explicit bound for $K$. We conjecture that $f$ is a local homeomorphism if $K_{I}(f)<2$. If $B_{f}$ contains a rectifiable arc, this is true by Martio $[12,6.6]$. However, $B_{f}$ need not contain any rectifiable arc. To see this, let $J$ be a quasiconformal curve in $R^{2}$ such that $J$ contains no rectifiable arc [11, p. 109], and let $g: \bar{R}^{2} \rightarrow \bar{R}^{2}$ be a quasiconformal mapping which maps $J$ onto a line $L$. By Ahlfors [2], $g$ can be extended to a quasiconformal mapping $g^{*}: \bar{R}^{3} \rightarrow \bar{R}^{3}$. If $h: \bar{R}^{3} \rightarrow \bar{R}^{3}$ is a winding mapping with $B_{h}=L$, then $f=h \circ g^{*}$ is a quasimeromorphic mapping with $B_{f}=J$.

4.8. Examples. For completeness we first construct an example of a quasiregular mapping of $R^{n}$ onto $R^{n}, n \geqq 2$, which is in the special case $n=3$ given in [21]. It shows that the local index has no upper bound in terms of the maximal dilatation and the dimension $n$.

4.9. Let $k$ be a positive even integer and set $Q_{1}=\left\{x \in R^{n}|| x_{i} \mid<1\right\}$. Given an integer $i, 1 \leqq i \leqq n$, let $J_{i}$ be the set of multi-indices $j=$ $\left(j_{1}, \ldots, j_{n}\right)$ such that $j_{i}$ is $-k$ or $k$ and $j_{m}$ is some of the numbers $-k, \ldots,-1,0,1, \ldots, k-1$ for $m \neq i$. For $j \in J_{i}$ let $C_{i j}$ be the $(n-1)$-dimensional cube $\left\{x \in R^{n} \mid j_{m} / k<x_{m}<\left(j_{m}+1\right) / k\right.$ if $m \neq i, x_{i}=$ $\left.j_{i} / k\right\}$. Let $\Phi_{k}$ be the set of sequences $D=\left(D^{1}, \ldots, D^{n-1}\right)$ where $D^{n-1}$ is some $C_{i j}$ and where $D^{p}$ is a $p$-dimensional face of the $(p+1)$-dimensional cube $D^{p+1}, p=1, \ldots, n-2$. For $D \in D_{k}$ let $T(D)$ be the $n$ dimensional simplex whose vertices are the vertices $y\left(D^{1}\right)$ and $z\left(D^{1}\right)$ of $D^{1}$, the centers $x\left(D^{p}\right)$ of $D^{p}, p=2, \ldots, n-1$, and the origin. Denote 
by $T^{\prime}(D)$ the $(n-1)$-dimensional simplex whose vertices are the vertices of $T(D)$ except the origin. We fix one such sequence $D_{0} \in \mathscr{D}_{k}$ such that $y\left(D_{0}^{1}\right)=e_{n}$ and $z\left(D_{0}^{1}\right)=e_{1} / k+e_{n}$.

We define a function $\beta$ of the vertices of the cubes $C_{i j}$ by setting for $u=\left(u_{1}, \ldots, u_{n}\right)$

$$
\begin{array}{ll}
\beta(u)=e_{n} & \text { if } k u_{1}+\ldots-k u_{n} \text { is even }, \\
\beta(u)=e_{1} / k+e_{n} \text { if } k u_{1}+\ldots+k u_{n} \text { is odd } .
\end{array}
$$

For $D \in \mathscr{D}_{k}$ let $g_{D}: R^{n} \rightarrow R^{n}$ be the affine homeomorphism which maps $T(D)$ onto $T\left(D_{0}\right)$ such that $g_{D}\left(y\left(D^{1}\right)\right)=\beta\left(y\left(D^{1}\right)\right), g_{D}\left(z\left(D^{1}\right)\right)=\beta\left(z\left(D^{1}\right)\right)$ ， $g_{D}\left(x\left(D^{2}\right)\right)=x\left(D_{0}^{2}\right), \ldots, g_{D}\left(x\left(D^{n-1}\right)\right)=x\left(D_{0}^{n-1}\right), g_{D}(0)=0$.

Next we define a quasiconformal mapping of the cone $E\left(D_{0}\right)$ onto the half space $H_{+}^{n}=\left\{x \in R^{n} \mid x_{n}>0\right\}$, where for $D \in \mathscr{S}_{k} E(D)=\left\{x \in R^{n} \mid x=\right.$ $\left.t z, z \in T^{\prime}(D), t>0\right\}$. Set $Y=\left\{x \in R^{n} \mid x_{n}=1\right\}$. Let $v \in Y$ and $r>0$ be such that $U=B^{n}(v, r) \cap Y$ is the maximal $(n-1)$-dimensional ball contained in $T^{\prime}\left(D_{0}\right)$. Let $\lambda$ be the radial projection of $V=U+\left(e_{n}-v\right)$ into $S^{n-1}$, i.e. $\lambda(z)=z /|z|$. Let $\alpha, 0<\alpha<\pi / 2$, be the angle between the $x_{n}$-axis $X_{n}$ and the line segments $[0, z], z \in S^{n-1}\left(e_{n}, r\right) \cap Y$. We define a mapping $w$ of the cone $W=\left\{x \in R^{n} \quad:=s z, z \in V, s>0\right\}$ of angle $\alpha$ onto $H_{+}^{n}$ as follows. For $x \in I \backslash X_{n}$ we set $w(x)=w\left(t, x^{\prime}, \vartheta\right)$ $=\left(t^{\tau / 2 \alpha}, x^{\prime}, \pi \vartheta / 2 \alpha\right)$ where we have used spherical coordinates such that $t=|x|, x^{\prime}=P_{n} x /\left|P_{n} x\right|$ where $P_{n}$ is the orthogonal projection onto $\partial H_{+}^{n}$, and $\vartheta$ is the angle between the $x_{n}$-axis and the line segment $[0, x]$. For $x \in W \cap X_{n}$ we set $w(x)=x_{n}^{\pi / 2 \alpha} e_{n}$. The required quasiconformal mapping $h: E\left(D_{0}\right) \rightarrow H_{+}^{n}$ is then obtained by setting $h(x)=w\left(t \lambda\left(\psi(z)+e_{n}-v\right)\right)$, where $x=t z, z \in T^{\prime}\left(D_{0}\right), t>0$, and where $\psi$ is the radial projection of $T^{\prime}\left(D_{0}\right)$ onto $U$.

Now we define a mapping $\varphi$ of the mion $\cup E(D)$ over all sequences $D \in \mathscr{T}_{k}$ by setting $\varphi(x)=s_{D}\left(h\left(g_{D}(x)\right)\right)$ for $x \in E(D)$ where $s_{D}$ is the identity mapping if $g_{D}$ is sense-preserving and the reflection in $\partial H_{+}^{n}$ if $g_{D}$ is sense-reversing. The mapping $\&$ can finally be extended continuously to a mapping $f_{k}: R^{n} \rightarrow R^{n}$. It is left to the reader to show that $f_{k}$ is quasiregular and that $K\left(f_{k}\right)$ has an upper bound which depends only on $n$ and not on $k$. On the other hand, $i\left(0, f_{k}\right) \rightarrow \infty$ as $k \rightarrow \infty$. We observe here also that $f_{k} \partial Q_{1}=\partial B^{n}$.

4.10. By slightly modifying the mappings $f_{k}$ in 4.9 for different $k$ we can construct a quasimeromorphic mapping $f$ with the property sup $i(x, f)=\infty$ as follows. For every positive integer $q$ we set $k_{q}=$ $2 \cdot 3^{q-1}$

$$
\Theta_{q}(x)=3^{q-1} x+\sum_{l=2}^{q} 4 \cdot 3^{l-2} e_{n} .
$$


and $Q_{q}=\Theta_{q}\left\{x \in R^{n}|| x_{i} \mid<1\right\}$. Then the cubes $Q_{q}$ are disjoint and $F_{q}=\bar{Q}_{q} \cap \bar{Q}_{q+1}$ is a face of $Q_{q}$. For every $q$ we first define mappings $\tilde{u}_{q}$ of $\bar{Q}_{q}$ by

$$
\tilde{u}_{q}(x)= \begin{cases}f_{k_{q}}\left(\Theta_{q}^{-1}(x)\right) & \text { if } q \text { is odd, } \\ v_{1}\left(v_{2}\left(f_{k_{q}}\left(\Theta_{q}^{-1}(x)\right)\right)\right) & \text { if } q \text { is even, }\end{cases}
$$

where $f_{k_{q}}$ is defined in 4.9, $v_{1}$ is the reflection in $S^{n-1}$, and $v_{2}$ is the reflection in $\partial H_{+}^{n}$. We first observe that those $(n-1)$-dimensional simplexes $\Theta_{q} T^{\prime}(D)$ and $\Theta_{q+1} T^{\prime}(\hat{D}), D \in \mathscr{D}_{k_{q}}, \hat{\bar{D}} \in \mathscr{D}_{k_{q+1}}$ (see 4.9), that are in $F_{q}$ coincide pairwise. We define a mapping $\chi_{q}: F_{q} \rightarrow F_{q}$ by letting $\chi_{q}(x)$ be the point in $\tilde{u}_{q+1}^{-1}\left(\tilde{u}_{q}(x)\right)$ which lies in $\Theta_{q} \bar{T}^{\prime}(D)$ if $x \in \Theta_{q} \bar{T}^{\prime}(D)$ and if $D \in \mathscr{D}_{k_{q}}$. Then $\chi_{q}(x)=x$ if $x$ belongs to the boundary of some simplex $\Theta_{q} T^{\prime}(D), D \in \mathscr{D}_{k_{q}}$. We shall modify the mappings $\tilde{u}_{q}$ so that the new mappings coincide pairwise in the sets $F_{q}$. To this end, we set for $q>1$ and $x \in \bar{Q}_{q}$

$$
u_{q}(x)= \begin{cases}\tilde{u}_{q}\left(t\left(\chi_{q-1}\left(\left(x-x_{q}\right) / t+x_{q}\right)-x_{q}\right)+x_{q}\right) & \text { if }\left(x-x_{q}\right) / t+x_{q} \in F_{q-1} \text { and } \\ \tilde{u}_{q}(x) \text { elsewhere, } & 0 \leqq t \leqq 1,\end{cases}
$$

where $x_{q}=\Theta_{q}(0)$ is the center of $Q_{q}$. The mappings $u_{q} \mid Q_{q}$ are quasimeromorphic, and $u_{q}$ and $u_{q+1}$ coincide in $F_{q}$. Furthermore, the maximal dilatation of $u_{q} \mid Q_{q}$ has an upper bound which depends only on $n$. The quasimeromorphic mapping $f_{0}: G_{0} \rightarrow \bar{R}^{n}, G_{0}=$ int $\left(\cup \bar{Q}_{q}\right)$, defined by $f_{0}(x)=u_{q}(x)$ for $x \in \bar{Q}_{q} \cap G_{0}$, has the required property sup $i\left(x, f_{0}\right)=\infty$.

4.11. As a final example we shall exhibit a discrete and open mapping $f: G \rightarrow R^{n}, n \geqq 3$, which is not topologically equivalent to any quasiregular mapping. Given a positive integer $p$, we define a mapping $\zeta_{p}$ of the cylinder $C=\left\{x \in R^{n} \mid x_{1}^{2}+x_{2}^{2}<2\right\}$ onto itself in cylinder coordinates (see 3.19) by

$$
\zeta_{p}(r, \varphi, z)=\left\{\begin{array}{l}
(r,(1+4 p)(\varphi-\pi / 4) \div \pi / 4, \theta) \text { if } \pi / 4 \leqq \varphi<3 \pi / 4, \\
(r, \varphi, z) \text { if } 0 \leqq \varphi<\pi / 4 \text { or } 3 \pi / 4 \leqq \varphi<2 \pi .
\end{array}\right.
$$

Let $\alpha_{p}$ be the translation $\alpha_{p}(x)=x+2(p-1) e_{1}$. We set

$$
G=\bigcup_{p=1}^{\infty} \alpha_{p} C^{\prime}
$$

and define $f: G \rightarrow R^{n}$ by $f(x)=\alpha_{p}\left(\zeta_{p}\left(\alpha_{p}^{-1}(x)\right)\right)$ for $x \in \alpha_{p} C$. Then $f$ is a sense-preserving, discrete, and open mapping with the property $i(x, f)=$ $p+1$ in the set $L_{p}=\left\{x \in R^{n} \mid x_{1}=2 p, x_{2}=0\right\}$. Suppose that there 
are homeomorphisms $g_{1}$ and $g_{2}$ such that $h=g_{1} \circ f \circ g_{2}$ is a quasiregular mapping. Then $h_{p}=h \mid g_{2}^{-1}\left(\alpha_{p} C\right)$ is quasiregular and $K\left(h_{p}\right) \leqq K(h)$. On the other hand,

$$
\inf _{x \in B_{h_{p}}} i\left(x, h_{p}\right)=p+1 .
$$

This gives by 4.2 a contradiction as $p \rightarrow \infty$.

In the plane the situation is different. Given a discrete open mapping $f: G \rightarrow R^{2}$, there exists by Stoillow's theorem [24, p. 120] an analytic function $\varphi$ and a homeomorphism $g$ such that $f=\varphi \circ g$. The following question remains open: Let $n \geqq 3$, let $f: G \rightarrow R^{n}$ be a discrete open mapping, and let $D$ be a subdomain in $G$ such that $\bar{D} \subset G$. Is $f \mid D$ topologically equivalent to a quasiregular mapping?

\section{The linear dilatations}

5.1. Upper bound for $H^{*}(x, f)$. It was proved in [13] that for a nonconstant quasiregular mapping $f: G \rightarrow R^{n}$ both $H(x, f)$ and $H^{*}(x, f)$ have upper bounds which depend on $K(f), n$, and $i(x, f)$. The result for $H^{*}(x, f)$ will now be sharpened to the extent that the upper bound does not depend on $i(x, f)$. This does not hold for $H(x, f)$.

5.2. Theorem. Let $f: G \rightarrow R^{n}$ be a non-constant $K$-quasiregular mapping and let $x \in G$. Then

$$
H^{*}(x, f) \leqq C^{*}(n, K)
$$

where $C^{*}(n, K)$ depends only on $n$ and $K$.

Proof. For $\quad r>0 \quad$ set $\quad U=U(x, f, r), l^{*}=l^{*}(x, f, r), L^{*}=$ $L^{*}(x, f, r), l=l\left(x, f, l^{*}\right), L=L\left(x, f, L^{*}\right), L_{l}=U(x, f, l)$, and $U_{L}=$ $U(x, f, L)$. There exists $r_{0}>0$ such that $U_{L}$ is a normal neighborhood of $x$ for $0<r \leqq r_{0}$. Fix such $r$. We may assume that $l<r<L$. Then $\left(U, \bar{U}_{l}\right)$ and $\left(U_{L}, \bar{U}\right)$ are ringlike condensers [13, 5.2]. Furthermore, $\partial U_{l}$ and $\partial U$ meet $S^{n-1}\left(x, l^{*}\right)$, and $\partial U$ and $\partial U_{L}$ meet $S^{n-1}\left(x, L^{*}\right)$. Therefore we have $\operatorname{cap}\left(U, \bar{U}_{l}\right)$, $\operatorname{cap}\left(U_{L}, \bar{U}\right) \geqq a_{n}$, where $a_{n}>0$ depends only on $n$. By $[13,6.2]$ we have

$$
\begin{aligned}
& \operatorname{cap}\left(U^{r}, \bar{U}_{l}\right) \leqq K_{o}(f) i(x, f) \omega_{n-1}\left(\log \frac{r}{l}\right)^{1-n}, \\
& \operatorname{cap}\left(U_{L}, \bar{U}\right) \leqq K_{o}(f) i(x, f) \omega_{n-1}\left(\log \frac{L}{r}\right)^{1-n} .
\end{aligned}
$$


On the other hand, we have by $[12,5.13,5.15]$

$$
K_{I}(f) \operatorname{cap}\left(U_{L}, \bar{U}_{l}\right) \geqq i(x, f) \operatorname{cap}\left(f U_{L}, f \bar{U}_{l}\right)=i(x, f) \omega_{n-1}\left(\log \frac{L}{l}\right)^{1-n}
$$

We also have

$$
\operatorname{cap}\left(U_{L}, \bar{U}_{l}\right) \leqq \omega_{n-1}\left(\log \frac{L^{*}}{l^{*}}\right)^{1-n}
$$

From (5.3) we get

$$
\left(\log \frac{L}{l}\right)^{n-1}=\left(\log \frac{L}{r}+\log \frac{r}{l}\right)^{n-1} \leqq 2^{n-1} K_{o}(f) i(x, f) \omega_{n-1} a_{n}^{-1} .
$$

Then the inequalities (5.4) and (5.5) imply

$$
\omega_{n-1}\left(\log \frac{L^{*}}{l^{*}}\right)^{1-n} \geqq \frac{a_{n}}{2^{n-1} K_{I}(f) K_{O}(f)} .
$$

Hence

$$
\frac{L^{*}}{l^{*}} \leqq \exp \left(2\left(\frac{\omega_{n-1} K^{2}}{a_{n}}\right)^{1 /(n-1)}\right)=C^{*}(n, K)
$$

The theorem is proved.

5.6. Example. Let $f_{0}: G_{0} \rightarrow \bar{R}^{n}$ be the quasimeromorphic mapping defined in 4.9 . If we compose $f_{0}$ with a stretching $\eta, \eta(x)=x_{1} e_{1}+\ldots+$ $x_{n-1} e_{n-1}+K x_{n} e_{n}, K>1$, then $g=f_{0} \circ \eta \eta^{-1} G_{0}$ is a quasimeromorphic mapping with the property $\sup H(x, g)=\infty$.

\section{Addendum}

When this manuscript was completed, two papers appeared partially overlapping with our work. Goldštein [7] obtains results related to 3.16. We have not been able to follow all details of his proofs. Poleckii [15] proves the path family inequality (2.13) for arbitrary quasiregular mappings and obtains results similar to 3.3 and 4.2 .

We have also received a preprint of Goldštein which contains a result similar to Theorem 4.6. 


\section{References}

1. Agard, S. and A. Marden: A removable singularity theorem for local homeomorphisms. - Indiana Math. J. 20 (1970), 455-461.

2. Ahrfors, L. V.: Extension of quasiconformal mappings from two to three dimensions. - Proc. Nat. Acad. Sci. U.S.A. 51 (1964), $768-771$.

3. Černavski, A. V. (Чернавский, А. В.): Конечнократные открытые отображения многообразий. - Mat. Sbornik 65 (1964), 357-369.

4. Church, P. T. and E. Hemmingsen: Light open maps on $n$-manifolds. - Duke Math. J. 27 (1960), 527-536.

5. Floyd, E.: Some characterizations of interior maps. - Ann. Math. 51 (1950), $571-575$.

6. Gehring, F. W.: Rings and quasiconformal mappings in space. - Trans. Amer. Math. Soc. 103 (1962), 353-393.

7. Goldštein, V. М. (Гольдштейн, В. М.): Одно гомотопическое свойство отображений с ограниченным искажением. - Sibirsk. Mat. Z̆. 11 (1970), 999-1008.

8. Hocking, J. G. and G. S. Young: Topology. - Addison-Wesley, 1961.

9. Hu, S.-T.: Homotopy theory. - Academic Press, 1959.

10. Hurewicz, W. and H. Wallman: Dimension theory. - Princeton University Press, 1941.

11. Lehto, O. and K. I. Virtanex: Quasikonforme Abbildungen. - Springer-Verlag, 1965.

12. Martio, O.: A capacity inequality for quasiregular mappings. - Ann. Acad. Sci. Fenn. A I 474 (1970), 1-18.

13. Martio, O., Rickman, S., and J. Väisälä: Definitions for quasiregular mappings. Ann. Acad. Sci. Fenn. A I 448 (1969), 1-40.

14. - - Distortion and singularities of quasiregular mappings. - Ann. Acad. Sci. Fenn. A I 465 (1970), 1-13.

15. Роцескі, Е. А. (Полецкий, Е. А.): Метод модулей дпя негомеоморфных квазиконформных отображений. - Mat. Sbornik 83 (1970), $261-272$.

16. Rado, T. and P. V. Reichelderfer: Continuous transformations in analysis. Springer-Verlag, 1955.

17. RešEtnjak, J. G. (Решетняк, Ю. Г.): Об устойчивости ғонформных отображений в многомерных пространствах. - Sibirsk. Mat. Ž. 8 (1967), $91-114$.

18. -»- Теорема Лиувигля при минимальных предположтенгях регулярности. Sibirsk. Mat. Ž. 8 (1967), 835-840.

19. -»- Отображения с ограниченным искажением как экстремали интегралов типа Дгрихле. - Sibirsk. Mat. Z̆. 9 (1968), 652-666.

20. Rickman, S.: Characterization of quasiconformal arcs. - Ann. Acad. Sci. Fenn. A I 395 (1966), $1-30$.

21. -»- Quasiregular mappings. - Romanian-Finnish seminar, Braşov 1969 (to appear).

22. Spanier, E. H.: Algebraic topology. - McGraw-Hill, 1966. 
23. SтоїLow, S.: Sur les transformations continues et la topologie des fonctions analytiques. - Ann. Sci. École Norm. Sup. 45 (1928), 347-382.

24. - - Leçons sur les principes topologiques de la théorie des fonctions analytiques. - Gauthier-Villars, 1938.

25. Твонтмс̌Uк, J. J. (Трохимчук, Ю. Ю.): О непрерывных отображениях областей әвклидова пространства. - Ukrain. Mat. Ž. 16 (1964), 196-211.

26. VÄrsÄL̈̈, J.: Lectures on $n$-dimensional quasiconformal mappings. - Van Nostrand Reinhold? (to appear).

27. Whyburn, G. T.: Analytic topology. - Amer. Math. Soc. Colloquium Publications, 1942.

28. ZoRIČ, V. A. (Зорич, В. А.): Теорема М. А. Јаврентьева о квазиконформных отображениях пространства. - Mat. Sbornik 74 (1967), 417-433.

29. -»- Изолированная особенность отображений с ограниченным искажением. Mat. Sbornik 81 (1970), 634-638.

University of Helsinki

Helsinki, Finland 